\title{
Bidirectional Interference Between Simulated Driving and Speaking
}

Kelsey Lynn Simmons

Brigham Young University

Follow this and additional works at: https://scholarsarchive.byu.edu/etd

Part of the Communication Sciences and Disorders Commons

\section{BYU ScholarsArchive Citation}

Simmons, Kelsey Lynn, "Bidirectional Interference Between Simulated Driving and Speaking" (2016). Theses and Dissertations. 6071.

https://scholarsarchive.byu.edu/etd/6071

This Thesis is brought to you for free and open access by BYU ScholarsArchive. It has been accepted for inclusion in Theses and Dissertations by an authorized administrator of BYU ScholarsArchive. For more information, please contact scholarsarchive@byu.edu, ellen_amatangelo@byu.edu. 
Bidirectional Interference Between Simulated Driving and Speaking

\author{
Kelsey Lynn Simmons
}

A thesis submitted to the faculty of

Brigham Young University in partial fulfillment of the requirements for the degree of

Master of Science

\title{
Christopher Dromey, Chair
}

Kristine Tanner

Shawn L. Nissen

Department of Communication Disorders

Brigham Young University

June 2016

Copyright (C) 2016 Kelsey Lynn Simmons

All Rights Reserved 


\author{
ABSTRACT \\ Bidirectional Interference Between Simulated Driving and Speaking \\ Kelsey Lynn Simmons \\ Department of Communication Disorders, BYU \\ Master of Science
}

The purpose of this study was to examine interference between concurrently performed speaking and driving tasks. Participants included 60 adults, 30 males and 30 females, with no history of communication disorders. They were divided into three different age groups of 20 participants each: younger (20s), middle-aged (40s), and older (60s). The participants were given a list of topics to consider and were instructed to select eight topics that they could talk about; they completed five practice trials of the driving simulator prior to the experimental recording to eliminate practice effects. Each participant completed the speaking task and driving task both separately and concurrently. The speaking task consisted of producing a monologue about the topics that they had selected. Dependent measures for speech included metrics relating to intensity, fundamental frequency, and the ratio of speaking to pausing time. The simulated driving task involved maintaining a constant speed and lane position on a freeway. Dependent measures for driving included metrics relating to speed, lane position, steering wheel position, and a count of steering wheel turns. Results indicated significant divided attention effects in speaking time ratio, intensity, speed, and steering wheel control. There was a significant age effect for intensity and fundamental frequency as the younger group had less variation with these variables compared to the other age groups. There was a significant age effect for lane position, steering wheel position, and speed as the younger group had less variation in lane and position compared to the other groups and the older group had more variation in speed and steering wheel position compared to the other groups. There was a significant gender effect for intensity and lane position as the females had less variation in intensity and more variation in lane position compared to the males. These findings suggest that divided attention conditions impact both speech and driving performance. The results also shed some light on the effects of age on concurrently performed speech and driving tasks. These findings imply that divided attention conditions should be incorporated in treatment to help patients generalize the skills learned in therapy to everyday communication.

Keywords: bidirectional interference, age, divided attention, acoustic speech measures, driving 


\section{ACKNOWLEDGMENTS}

I would like to thank my thesis advisor, Dr. Christopher Dromey, for his expertise, feedback, support, and mentoring over the course of this research as well as my education. His enthusiasm and support gave me the motivation to complete a project that would be rewarding to me, and I thank him for this experience.

I would also like to thank those that helped make this project possible: Garrett Porter for programming the driving simulator, Katy Glenn for helping with data collection, and to the many participants who sacrificed their time to this research. I am also grateful to my committee for their guidance, time, and support in constructing this thesis. I am also beyond grateful for the support and friendship of my cohort throughout graduate school. They made these past two years of my education not only possible, but also enjoyable.

Many thanks are also due to my family for their constant encouragement and support as I pursued a master's degree. Their support motivated me to push through difficult times and helped me achieve what I thought was impossible. Most of all, I would like to thank my husband for the sacrifices he has made to help make this dream of mine possible. His patience and continual love and support have been invaluable to me throughout this journey and I would not have been able to accomplish this without him. 


\section{TABLE OF CONTENTS}

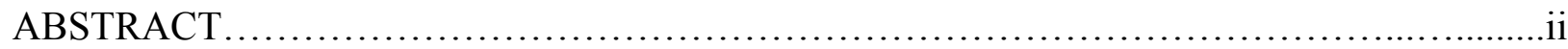

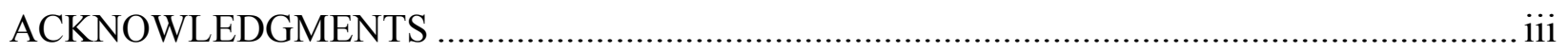

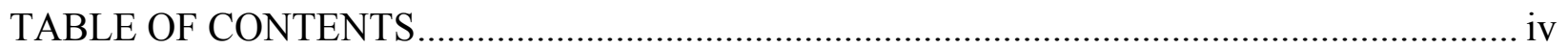

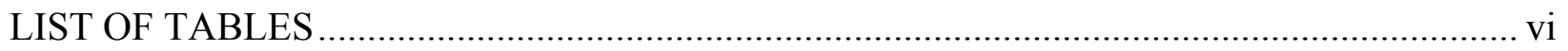

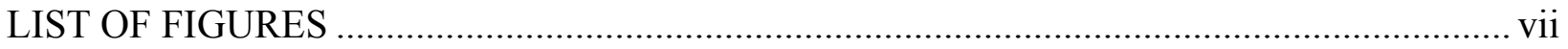

DESCRIPTION OF THESIS CONTENT …................................................................. viii

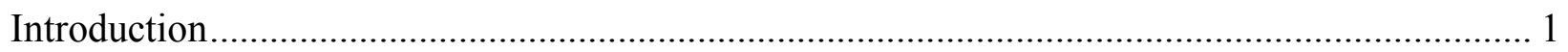

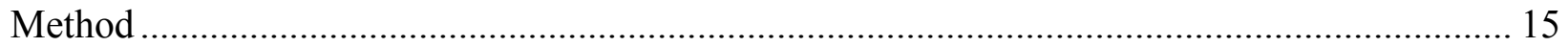

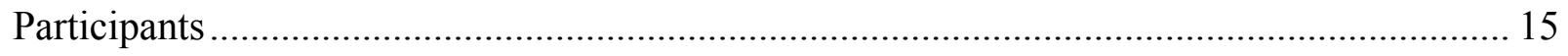

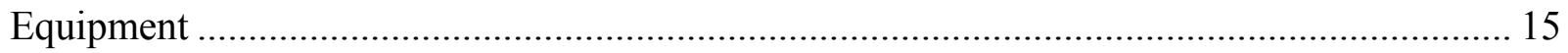

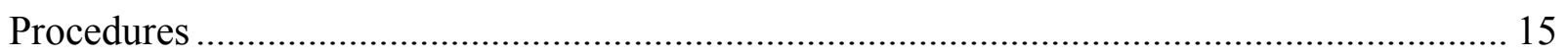

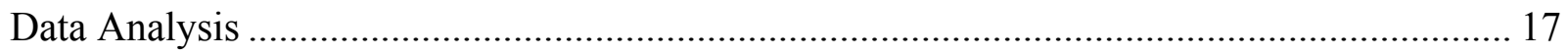

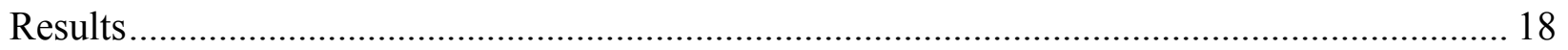

Divided Attention Effects..................................................................................... 22

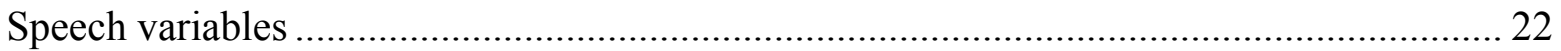

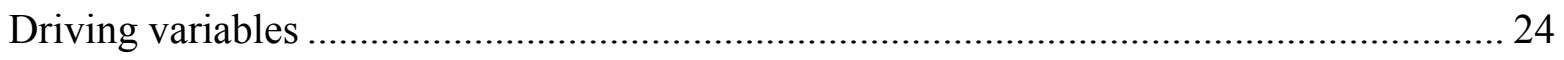

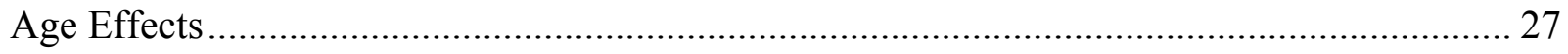

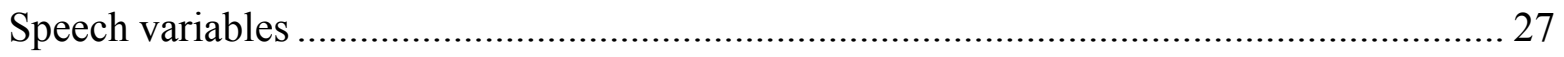

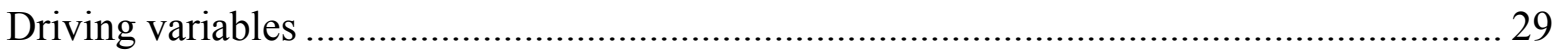

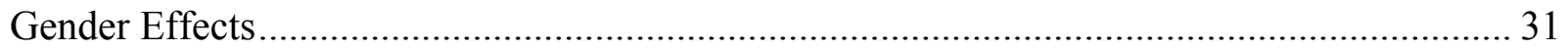

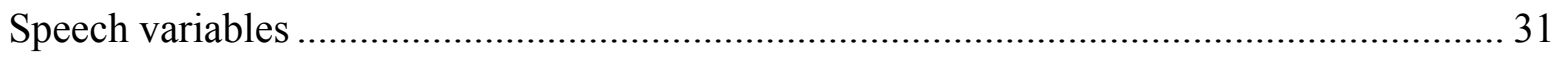

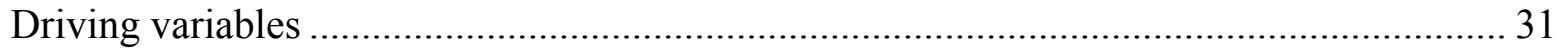




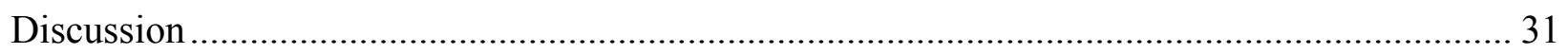

Effects of Driving on Speech Performance........................................................................... 32

Effects of Speaking on Driving Performance …………….................................................. 34

Limitations of the Current Study and Directions for Future Research ................................... 37

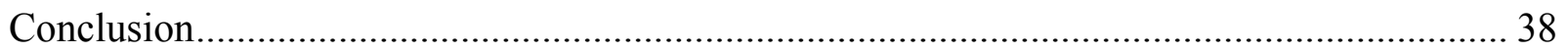

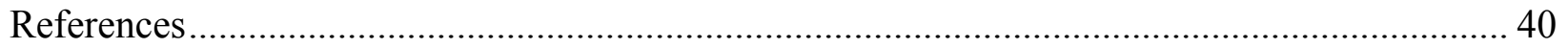

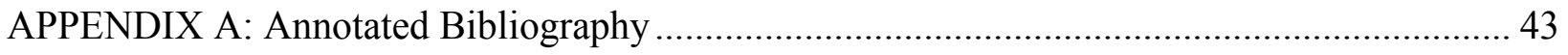

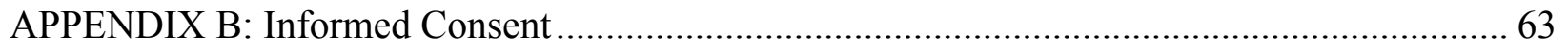

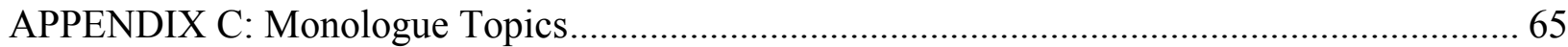




\section{LIST OF TABLES}

Table $1 \quad$ Descriptive Statistics for Average Speaking Time Ratio ..................................... 19

Table 2 Descriptive Statistics for Average Intensity (dB SPL at $50 \mathrm{~cm}$ ) .......................... 20

Table 3 Descriptive Statistics for Standard Deviation of Intensity in $d B$........................ 20

Table 4 Descriptive Statistics for Standard Deviation of Fundamental Frequency

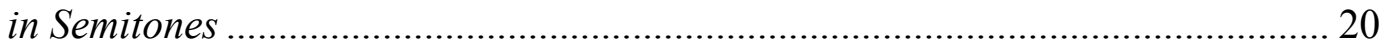

Table 5 Descriptive Statistics for Standard Deviation of Lane Position

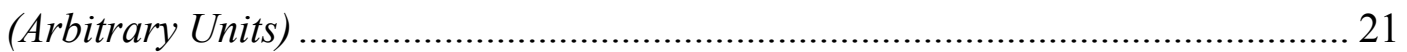

Table $6 \quad$ Descriptive Statistics for Average Speed in km/h .......................................... 21

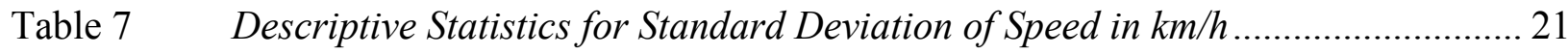

Table 8 Descriptive Statistics for Standard Deviation of Steering Wheel Position

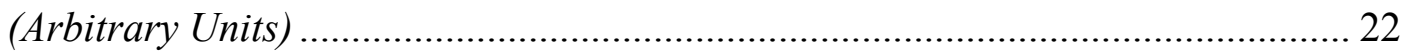

Table 9 Descriptive Statistics for Average Number of Steering Wheel Turns ................... 22 


\section{LIST OF FIGURES}

Figure $1 \quad$ Mean (and 95\% confidence interval) of the speaking time ratio ....................... 23

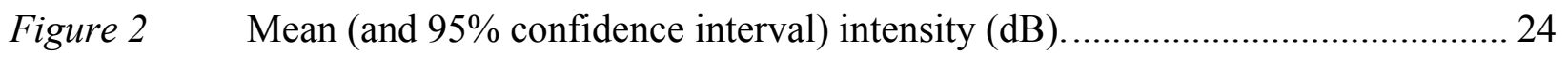

Figure 3 Mean (and 95\% confidence interval) of the standard deviation of speed

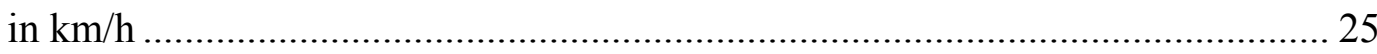

Figure $4 \quad$ Mean (and 95\% confidence interval) of standard deviation of steering wheel

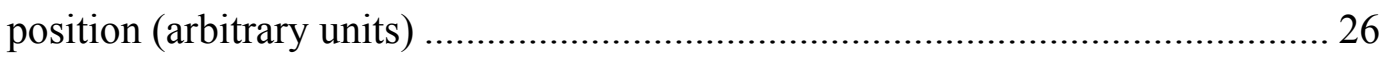

Figure $5 \quad$ Mean (and 95\% confidence interval) number of steering wheel turns ............... 27

Figure 6 Mean (and 95\% confidence interval) standard deviation of intensity (dB) ........ 28

Figure $7 \quad$ Mean (and 95\% confidence interval) standard deviation of fundamental frequency

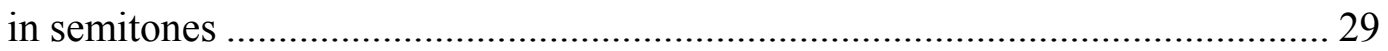

Figure 8 Mean (and 95\% confidence interval) standard deviation of lane position

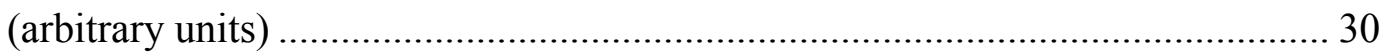




\section{DESCRIPTION OF THESIS CONTENT}

This thesis, Bidirectional Interference Between Simulated Driving and Speaking, is the result of a research project and portions of this thesis may be published as part of articles listing the thesis author as a co-author. The body of this thesis is written as a manuscript suitable for submission to the university. An annotated bibliography is presented in Appendix A and an informed consent is presented in Appendix B. The list of monologue topics used for the speech task is included in Appendix C. 


\section{Introduction}

Successful human communication involves coordinating multiple processes: language formulation, intonation, speech rate, and the fine motor skills of articulation. With the many processing systems that contribute to communication, attentional resources are required to successfully execute these functions. Seldom, however, is human communication conducted in isolation, as people are typically engaged in other activities during conversations (e.g., walking or driving). While multi-tasking in speech is considered normal, the attentional demands of performing two tasks simultaneously can create interference that can cause a decline in performance in one or both tasks. Although these changes in speech performance can be subtle, recent studies have shown that this interference can even be found in individuals without speech or language disorders (Bailey \& Dromey, 2015; Dromey \& Bates, 2005).

Driving is a common activity that requires a division of attention in managing lane maintenance, speed control, navigation, following distance, etc. With technology advancing every year, cell phones have increased in popularity, with over $90 \%$ of Americans having a cell phone as of 2010 (Strayer, Watson, \& Drews, 2010). Consequently, driving while concurrently talking on a cell phone has become an activity that many people participate in daily, with $85 \%$ of drivers claiming to engage in cell phone use while driving a vehicle (Strayer et al., 2010). However, due to the competing attentional demands of both driving and conversing, studies have shown a high correlation between accident rates and in-vehicle cell phone use, because conversing on a cell phone increases drivers' likelihood of an accident by four times (Beede \& Kass, 2006; Cao \& Liu, 2013; Strayer \& Johnston, 2001; Strayer et al., 2010). In fact, as of 2010 , it was estimated that $28 \%$ of all vehicle accidents were caused by cell phone use (Strayer 
et al., 2010). Thus, the advent of the cell phone has led to a substantial increase in risk to drivers' safety.

Over the past few decades, cognitive psychologists have dedicated many research studies to understanding attentional processes, specifically how individuals can concurrently carry out multiple tasks and how much these tasks interfere with each other. Two theories are commonly recognized in the field of divided attention: structural and capacity. Structural theories propose that certain cognitive processes or mental operations are carried out in a sequential order, and that in dual-task conditions, a bottleneck arises as the attentional processes are occupied with the first task before attending to the second task. As a result, performance in the second task becomes delayed (Pashler \& Johnston, 1998; Wickens, 1981). Based on this model, Wickens suggested that a bottleneck can arise in any stage of processing, and that it is not limited to just one stage or one mental process. In contrast, capacity theories suggest that the brain has limited cognitive resources, and as a result, dual-task performance suffers as one task may demand more attentional resources than the other (Pashler \& Johnston, 1998; Strayer et al., 2010; Wickens, 1981). Thus, the rate or efficiency of the processing depends on how many resources are available to complete the task at any given time (Pashler \& Johnston, 1998). Wickens also outlined structural and capacity theories in his publications. However, the author raised the possibility of a time-sharing model, and suggested that attention can rapidly alternate between two tasks in dual-task situations. He proposed that if the alternation of attention is completed smoothly and rapidly enough, performance in both tasks could remain similar.

Cognitive psychologists have also questioned whether the type and extent of interference in dual-tasks depends on the areas in the brain that are responsible for carrying out the two tasks; if they are anatomically closer together, the interference may be greater; this is known as the 
functional distance hypothesis (Bailey \& Dromey, 2015; McDowd \& Craik, 1988). Similar to this theory, researchers have also raised the possibility that when the processes that are needed to perform the two tasks are similar (e.g., auditory, visual, motor), a crosstalk effect can occur, resulting in impaired performance in one or both tasks (Pashler \& Johnston, 1998). Bergen, Medeiros-Ward, Wheeler, Drews, and Strayer (2013) conducted a dual-task study to determine the nature of interference when individuals concurrently drive and talk on a cell phone. The study focused on whether the interference could be explained by the cross-talk hypothesis (also known as the domain-specific interference theory) or the limited capacity theory (also known as the domain-general interference theory). The experiment consisted of a driving task and four different language conditions (control, visual, motor, and abstract) to observe the effects of type of language activity on driving. Each language condition consisted of sentences with content that corresponded to the type of language processing (e.g., "The letters on a stop sign are red" for the visual condition). The participants were instructed to respond to the sentences by stating whether they were true or false. Vehicle following distance measures revealed that the motor and visual conditions yielded greater and more variable following distances compared to the other conditions. The visual and abstract language conditions were most affected by the dualtask condition as the participants' responses became less accurate. These findings suggest that a code conflict can exist when individuals are using perceptual and motor language when concurrently performing perceptual or motor tasks; this provides support for the crosstalk hypothesis.

Although distraction can cause a decline in driving performance, many distracted drivers arrive at their destinations without driving off the road or causing an accident. In fact, previous studies have found a positive correlation between lane maintenance and cognitive workload: as 
cognitive workload increases, lane maintenance increases as well (Becic et al., 2010; Beede \& Kass, 2006). Recently, researchers have theorized two loops of cognitive control that underlie skilled performance to explain this phenomenon: the outer loop and the inner loop. The outer loop is governed under attentional control, and can be brought into conscious awareness, whereas the inner loop is automatic and operates without conscious awareness (Medeiros-Ward, Cooper, \& Strayer, 2014). Thus, when learning a new skill, the outer loop is used to learn the skill as it has a higher level of control and the individual is aware of their performance. As individuals become more competent with the task, the inner loop takes on a greater role as the skill requires less control and effort. The higher level of control is then relied on when the task environment becomes unpredictable, and the individual becomes more consciously aware of their performance (Medeiros-Ward et al., 2014). This theory is known as the hierarchal control theory (HCT) and it claims that (a) performance that is dependent on the outer loop will get worse when less attention is given to the task, and (b) performance that is dependent on the inner loop will get worse when more attention is given to the task (Medeiros-Ward et al., 2014).

To assess this theory, Medeiros-Ward et al. (2014) had participants perform tasks under three different levels of cognitive workload while concurrently driving. Three levels of wind entropy (low, medium, and high) were also incorporated to make driving routines unpredictable. The researchers found that an increase in cognitive workload was associated with an improvement in lane maintenance, but that an increase in cognitive workload in addition to unpredictable driving conditions (higher levels of wind entropy) caused more variability in drivers' lane positioning. These findings suggest that distracted drivers may not drive off the road in predictable driving situations, because lane maintenance is a skill within the inner loop of control that can successfully be executed without conscious attention. However, in unpredictable 
driving scenarios, a higher level of control is needed to stay in the lane, supporting the HCT model. Similar results were found in another study, as participants stayed in their lane more often in the dual-task condition compared to the single-task condition (Kubose et al., 2006). The authors, however, attributed these results to drivers prioritizing certain tasks and making adjustments to adapt to their driving environment.

Other researchers claim that these findings could be explained by four uncontrolled factors in the experiments: strategic tradeoff of attentional resources between different tasks, the level of difficulty for lane keeping, the lack of cognitive task performance measures, and participant motivation (Cao \& Liu, 2013). In their own study, Cao and Liu investigated the effects of speech comprehension tasks on concurrent lane keeping and found that although the secondary speech task did not immediately impact lane keeping performance, speech comprehension performance declined due to the increased cognitive workload and if both tasks continue to be performed over a longer period of time, driving performance can eventually decline as well. These results contradict the findings in other studies (Medeiros-Ward et al., 2014; Kubose et al., 2006).

Researchers have also raised the possibility that because cell phone conversations occupy some of the drivers' attention, drivers fail to attend to their driving environment even if their eyes are on the road (Strayer et al., 2013; Strayer \& Drews, 2004, 2007; Strayer et al., 2010). Researchers refer to this phenomenon as inattention blindness and suggest that cognitive and visual distractions may be linked (Strayer et al., 2013). Strayer and Drews (2007) conducted four studies to determine if this hypothesis could explain the effects of conversing while driving. To do so, the authors compared memory tests after performance in each condition (single-task and dual-task), and examined the P300 component's amplitude in electroencephalographic 
recordings to measure the amount of attention and the level of task difficulty. In the fourth study, the authors compared the attentional demands between a conversation with a passenger in the vehicle, and a conversation on a cell phone by comparing navigation success rates. When compared to the single-task condition, the first two studies revealed that the participants recognized half as many objects in the dual-task condition, even if their eyes were fixated on the objects for the same amount of time as in the single-task condition. The third study revealed that the P300 component's amplitude decreased by $50 \%$ in the dual-task condition compared to the single-task condition, suggesting that the attentional demands of the conversation caused a decrease in the amount of brain activity directed towards the driving environment. The fourth study revealed that the attentional demands were less for the passenger conversation compared to the cell phone conversation. Based on all the studies, conversing on a cell phone requires enough attentional demands that drivers fail to attend to some objects in their driving environment, and as a result of this inattention blindness, drivers create riskier driving situations (Strayer et al., 2013; Strayer \& Drews, 2007; Strayer et al., 2010).

Although these studies provide support for the notion of cognitive sources of distraction in the automobile, Strayer et al. (2010) raise the question of whether or not driving impairment could be caused by manual or visual interference. Strayer and Johnston (2001) examined this topic by narrowing down the source of the interference, whether it be due to peripheral interference (i.e., holding the phone) or the attentional interference created by the conversation. The participants were divided into three groups: radio control, handheld phone, and hands-free phone. The radio control group was instructed to listen to the radio while driving, while the two phone groups participated in two different tasks: a repeating task and a word generator task. The word generator condition yielded more tracking errors than the repetition condition when 
simultaneously driving, suggesting that generating new words creates more interference and requires more attentional resources than repeating words. However, both phone conditions revealed an increase in missed traffic signals and delayed reaction times, indicating that peripheral factors do not significantly contribute to the interference found in driving dual-task situations. Thus, the manipulation of the cell phone, or manual interference, does not fully explain the interference found in dual-task driving scenarios, as found in previous studies ( Strayer et al., 2013; Strayer \& Drews, 2004).

As would be expected, visual interference while driving creates riskier driving situations as the driver is not as fully focused on the driving environment. Beede and Kass (2006) had drivers participate in two tasks while driving: a signal detection task and a cell phone conversation task. The signal detection task had participants press the buttons on the steering wheel that corresponded to the signal arrows that appeared in the bottom corners of the screen. Both the cell phone and signal detection tasks resulted in an increase in the number of attention lapses committed while driving, but the cell phone task had a significant impact on attention as participants committed more traffic violation when conversing on a cell phone than in conditions without cell phone usage. These results suggest that although visual interference is a source of distraction in a vehicle, cognitive tasks, such as talking while driving, demand more attentional resources and as a result, these tasks can have greater impacts on driving performance.

Other studies have focused on the impact of different aspects of communication, such as speech production and speech comprehension, to determine the type of speech task that causes the most interference in driving. Studies have shown that both aspects of communication have similar effects on driving performance, and thus, both speech production and speech comprehension create interference when it comes to driving (Kubose et. al, 2006). In another 
study, Dula, Martin, Fox, and Leonard (2011) found that even the type of conversation affects driving performance differently, as more "emotional" phone calls have a greater impact on driving compared to "mundane" phone calls due to an increase in cognitive workload in emotional phone calls. Other studies have also shown that driving behaviors are influenced by whether the conversation was with a passenger in the vehicle or on a cell phone as the attentional demands differ between the two types of conversations (Strayer \& Drews, 2007). For instance, Drews, Pasupathi, and Strayer (2008) found that drivers demonstrated more difficulty in navigation and lane maintenance in the cell phone condition compared to the passenger condition, and that the conversation in the passenger condition had more references to the traffic and turn taking. However, production rate in the passenger condition was less than the cell phone condition. In a follow-up study, Strayer et al. (2013) found that when the passenger could not help with the driving task, the two different types of conversations had minimal differences. Based on these studies, it can be inferred that driving performance is not only affected by conversations, but the type of conversation can amplify these deficits in driving.

Based on the reviewed research, it is evident that conversations in a vehicle impact driving performance. However, because the effects on communication are not as potentially dangerous as the effects on driving, most research has neglected to study the impact of driving on communication. Becic et al. (2010), however, did examine speech measures to determine how driving impacts talking. They found that along with conversations interfering with driving performance (i.e., slowed velocity, delayed braking reaction times, and better lane maintenance), driving had a negative impact on speech production, comprehension, and memory as drivers had better recall of stories and more accurate story retelling during the speech-only task compared to 
the dual-task condition. Accuracy in story recall was also impacted when the driving route was more difficult (e.g., driving through intersections).

Although research on speech measures in dual-task driving situations is limited, multitasking in speech is the norm, not the exception. Thus, several studies have examined how speech is affected when simultaneously performing different types of tasks outside of driving. For instance, Dromey and Bates (2005) conducted a study to examine the bidirectional interference of speech and nonspeech tasks by having participants repeat Peter Piper would probably pick apples while concurrently performing linguistic, cognitive, and visuomotor tasks. Each task was performed individually in a single-task condition, then in a dual-task condition which combined the speech task with a nonspeech task. The results of these conditions revealed that certain combined conditions had a greater impact on speech and nonspeech performance than other conditions. For instance, the combined linguistic task impacted speech performance by increasing the lower lip spatiotemporal index (STI: more variable labial movements) and sound pressure level (SPL: louder speech). This combined task also impacted nonspeech performance as participants had lower linguistic scores and more latencies in their responses compared to the linguistic-only condition. Although the combined cognitive condition had similar results for nonspeech task performance, speech performance remained unchanged in this condition. The combined visuomotor condition, however, revealed an impact on speech performance as utterance duration and lower lip/jaw displacement decreased. However, performance on the visuomotor task remained unchanged. Thus, different types of tasks impact speech performance differently, and the interference in dual-task conditions can be bidirectional or unidirectional. The task that impacted speech the most, however, was the linguistic task, which supports the functional distance hypothesis. 
In another study, Oomen and Postma (2001) examined the influence of divided attention tasks on disfluencies (filled pauses and repetitions) that are commonly produced in typical speech. The two tasks in the study included a story-telling task and a blind tactile-form recognition task, both of which were performed separately and then together for a divided attention condition. The researchers found a decline in performance in both tasks as the participants had more pauses and repetitions (specifically sound/part-word and word repetitions) and poorer performance in the tactile recognition task in the divided attention condition compared to the single-task conditions. Thus, the results provide support that speech tasks can also have an impact on nonspeech task performance.

Although the attentional demands in divided attention tasks can cause interference in task performance, a small percentage of individuals can perform multiple tasks at once without impairment, whom researchers have referred to as supertaskers (Strayer et al., 2010). While some individuals thrive in divided attention situations, others tend to struggle, especially older adults. Due to the cognitive changes that arise from aging, researchers have recently become more interested in studying dual-task performance across the adult life span. A consistent finding in previous studies is that older adults have poorer performance when performing dualtasks (Bailey \& Dromey, 2015; Becic et al., 2010; McDowd \& Craik, 1988). There are many theories as to why this decline in performance happens. One theory is that as the brain ages, the cortical lining of the brain thins, causing the areas of operation to be closer together. Thus, more interference is created when trying to perform both tasks at once, as claimed in the functional distance hypothesis (McDowd \& Craik, 1988). Other theories propose that aging in the brain causes either a decline in short-term memory and storage capacity, or that the processing resources in older adults are limited in tasks that require a division in attention (McDowd \& 
Craik, 1988). Beyond these theories, researchers are still unclear as to why older adults have more difficulty in divided attention situations.

McDowd and Craik (1988) conducted two experiments to examine how task complexity interacts with age to impact dual-task performance. Participants in the first experiment completed an auditory and visual task, both of which were performed at two levels of difficulty: easy and hard. The second experiment also involved two tasks with two levels of difficulty. Experiment one revealed that younger and older adults were both affected by task complexity as reaction times increased for both groups when the task increased in complexity. The older adults, however, had more errors under the dual-task condition compared to the younger adults, and these decrements in dual-task performance were amplified when the task became more difficult. Although this experiment yielded mixed results, the researchers still found age to have a negative effect on dual-task performance.

This decline in dual-task performance in older adults is also seen with their driving behaviors. In fact, recent statistics show that driving fatality rates decline from young-adult to middle-aged years, but then increase steadily as the aging process continues, even though older adults have more driving experience and take fewer risks compared to younger drivers (Strayer $\&$ Drews, 2004). Consequently, when cell phone conversations take place in the vehicle, these individuals have to prioritize, because it is difficult for the aging brain to complete both tasks successfully at the same time. For instance, Becic et al. (2010) examined the effects of aging and concurrent driving and speech tasks. Driving measures showed that the older adult drivers slowed down and stayed in their lane better under dual-task conditions compared to the younger adult drivers. The researchers also reported that the older-aged group moved slowly through intersections and braked further away from stop signs compared to the younger group. Even 
with these driving adjustments, the older adults performed worse overall on the speech tasks (story retell and recall). Although the driving behaviors seemed safer in the older adults, the results of the speech tasks revealed that these individuals demonstrated more difficulty under dual-task conditions compared to the younger adults, and thus, to maintain safety, the older adult drivers allowed their speech performance to suffer to pay more attention to their driving. Despite these findings, in another study, Strayer and Drews found age not to be a significant factor in dual-task driving scenarios, as both older and younger adult drivers were impacted by the dualtask condition equally. A limitation in this study, however, is that the variety of topics used in the cell phone conversations was not consistent, and thus, the cognitive workload may have varied.

Although the research is limited, some studies have investigated the effects of aging on speech performance. Ballard, Robin, Woodworth, and Zimba (2001) examined differences in articulatory motor control across different ages through lower lip, jaw, and laryngeal movement measures during a tracking task. The correlation between the target and the individual's tracker signal improved after 8 years of age until 15 to 20 years of age, when the correlation plateaued. Following this plateau was a steady decline in correlation at 40 to 45 years of age. The average amplitude difference between the two signals was also lowest for those adults between 17 and 45 years of age, showing that the older-aged adults varied in articulatory tracking performance more than the younger- and middle-aged adults. This pattern was found on all other measures, indicating that the effects of aging impact articulatory performance on visuomotor tracking tasks.

Bailey and Dromey (2015) also conducted a study to investigate the effects of aging and the bidirectional interference between a speech task and nonspeech tasks (linguistic, cognitive, and manual motor). Participants included three different age groups: younger-aged, middle- 
aged, and older-aged. The speech task involved repeating the sentence I saw Patrick pull a wagon packed with apples every time participants heard a tone. The dual-task condition consisted of the speech task combined with one of the nonspeech tasks. Dual-task conditions yielded a decline in speech performance across all age groups as the spatiotemporal index (STI) of the lower lip increased in all the combined task conditions. The linguistic and cognitive combined tasks resulted in an increase in sentence duration, as well as a significant decrease in upper lip and lower lip coordination, as reflected in a correlation measure. The manual motor combined task resulted in reduced lower lip displacement and significantly increased SPL. Nonspeech task performance was also affected as the number of correct and total responses decreased in the linguistic and cognitive combined conditions compared to their isolated counterparts. Among these findings, the older-aged group had longer utterance duration in the speech task and poorer performance on all variables in the nonspeech tasks. These results indicate that task type and age affect speech performance during dual-task conditions, and that age can have a significant effect on speech and nonspeech task performance, which supports previous findings.

Although these studies investigated the interference arising when simultaneously performing speech and nonspeech tasks, the speech tasks in many of these earlier studies lacked ecological validity, because the participants were instructed to repeat the same phrase or sentence over and over again. Although these tasks allow researchers to directly compare the same words under different conditions, the tasks do not reflect the way speech is produced in everyday life. It has also been recognized that talking has dangerous effects on driving, but not enough research has been dedicated to how driving impacts the way speech is produced, even though previous studies have shown that conversations create interference while driving. Strayer et al. (2013) 
even quantified the amount of cognitive distraction of various activities that take place in the vehicle and found that passenger and cell phone conversations were ranked in the second highest category of cognitive distraction. Thus, the division of attention that takes place when driving and talking on a phone creates interference, although only driving measures have been extensively examined.

Based on previous research, it is hypothesized that all participants will have poorer performance in both tasks when under dual-task conditions compared to single-task conditions, and that the older adults will have a greater decline in performance compared to younger adults. Acoustic measures of connected speech, including the average and variability of fundamental frequency $\left(\mathrm{F}_{0}\right)$ and intensity, as well as the ratio of speaking to pausing time, will be used to quantify speech performance. A predicted decline in speech performance would be shown by a decrease in intensity and time spent speaking, as well as reduced variability in intensity and $\mathrm{F}_{0}$. Driving measures including speed control, steering wheel turns and position, and lane maintenance will be used to quantify driving performance. Poor driving performance would be reflected by a reduction in average speed, an increase in the number of steering turns, as well as an increase in variability of lane maintenance, steering wheel position, and speed. It is important to understand the bidirectional interference between simultaneous tasks, as it can provide a deeper understanding of the consequences of divided attention. Since speaking is common under dual-task conditions, speech performance measures can provide therapists with insight as to how to make the clinic environment more representative of real-life talking situations, which in turn, can help clients in the clinic to be more robust when they apply what they learn to their daily lives. 


\section{Method}

\section{Participants}

Thirty men and thirty women participated in the study, divided evenly into three age groups: young adults (ages 20-30, $M=23.10, S D=2.56$ ), middle-aged adults (ages 40-50, $M=$ 45.70, $S D=2.81$ ), and older adults (ages 60-71, $M=65.60, S D=3.80$ ). All participants were native speakers of English, had no prior history of speech, language, or hearing disorders, had normal or corrected-to-normal vision, had functionally normal communication, and had a valid driver's license. Each participant signed informed consent documents approved by the Institutional Review Board prior to participation in the study.

\section{Equipment}

Each participant was seated in a sound booth to provide an optimal environment to make high quality acoustic recordings and to reduce possible auditory distractions. A microphone headset was used to acquire the participant's speech, which was recorded digitally to a laboratory computer with Audacity software (version 2.0.6; http://audacity.sourceforge.net/). Prior to data collection, the headset was calibrated using a sound level meter (Extech 407736) $50 \mathrm{~cm}$ away from the speaker. OpenDS software (version 3.5; https://www.opends.eu/) was used to record driving performance as subjects used a Logitech Driving Force GT steering wheel and gas/brake pedal interface on a lab computer to navigate a virtual road. The lab computer had a 24-inch display to provide a view of the simulated driving environment for the participants.

\section{Procedures}

All participants completed a driving task and a speech task, each performed separately in an isolated condition, and together simultaneously in a divided attention condition. In a pilot study, an unfamiliar user completed the driving task 10 times. Based on the recordings from 
these 10 trials, it was found that the $S D$ of lane position and speed plateaued around the fifth trial, indicating that 15 minutes of practice was enough time for users to become familiar with the driving simulator. Therefore, each participant completed five practice trials with the driving simulator prior to recording data to familiarize themselves with the software and to reduce the impact of possible practice effects during the experiment.

As participants progressed through the practice trials, the instructions gradually became more specific until the participants were practicing the actual driving task. On the first two trials, the participants were instructed to familiarize themselves with the simulator during a freeway driving activity, and to take the first exit. On the third trial, they were instructed to try to maintain a speed of $100 \mathrm{~km} / \mathrm{h}$ in addition to the previous instructions. The fourth trial required participants to stay in the center of the right lane in addition to maintaining their speed. The fifth practice trial had the same requirements as the previous trial.

All participants were given several minutes to consider monologue topics from an extended list, and were instructed to choose eight topics of interest (see Appendix C for the list of topics). Once the experiment began, each participant was presented with a topic that they had chosen, and they were instructed to talk about it. If they ran out of things to say, they would say "next," and the experimenter presented a new topic for them to respond to. The experimenter continued to present topics to the participant until the recording was about $80 \mathrm{~s}$ long to ensure it included at least $60 \mathrm{~s}$ of the participants' speech for analysis.

The driving task consisted of participants driving a specific course ("Motorway") using the OpenDS software. The duration of the task was approximately two minutes, with a travelling distance of $1300 \mathrm{~m}$ on a freeway. The course consisted of merging onto and later exiting a twolane freeway. They were also instructed to merge onto the freeway as soon as they reasonably 
could, stay in the center of the right lane after they merged onto the freeway, maintain a speed of $100 \mathrm{~km} / \mathrm{h}$, and take their first exit. The simulation included other vehicles on the freeway, which traveled at a fixed speed of $100 \mathrm{~km} / \mathrm{h}$, in order to provide additional distraction to the participants. During the divided attention condition, participants drove the simulator while they completed the speech task. The three different conditions were all performed in a randomized order for each participant. The total time to complete all practice and experimental tasks was about 35 minutes per participant.

\section{Data Analysis}

Speech measures in the isolated condition were compared with the same variables in the divided attention condition to quantify the impact of driving on speaking. Speech recordings were analyzed using the Praat software program (version 5.4; Boersma \& Weenink, 2014). Experimenter speech, pauses in between topics, and nonspeech behaviors (laughing, coughing, etc.) were trimmed from the recordings prior to analysis. Once trimmed, the middle $60 \mathrm{~s}$ of the speech from each condition were used for analysis.

Acoustic measures of connected speech, including patterns of $\mathrm{F}_{0}$ and intensity, as well as the proportional amount of time participants talked during their responses, were computed to quantify speech performance. $\mathrm{F}_{0}$ was measured by taking the $M$ and $S D$ in the 60 -second recording. The $\mathrm{F}_{0}$ range was adjusted in Praat to avoid tracking errors. The voicing report from this program provided with the $M$ and $S D$ in $\mathrm{F}_{0}$. Because the $\mathrm{F}_{0}$ range differs between males and females, the $S D$ values were converted into semitones by using a spreadsheet equation. Intensity was also measured by taking the $M$ and $S D$ of the 60 -second recording. To avoid recording the intensity level of pauses or nonspeech sounds, a dB floor was selected based on the level of intensity of the softest speech sounds in the recording. The intensity listing in Praat was 
exported as a comma separated values file (csv). The csv file was then brought into a custom Matlab application (version 9.0; The Mathworks, Inc., 2016) to compute the $M$ and $S D$ of intensity above the selected floor. The speaking time ratio was expressed as a proportion, 1.0 would be all speaking and no pausing, 0.75 would be $75 \%$ speaking and $25 \%$ pausing, etc. A custom Matlab application was used to compute the speaking and pausing ratio, which recognized pauses as being longer than $200 \mathrm{~ms}$. The application also normalized the intensity of the file to 100 . Ten percent of the normalized max was selected as the threshold, above which was operationally defined as speaking, and below which was defined as pausing.

Driving measures in the isolated condition were compared with those in the divided attention condition to quantify the impact of speaking on driving. Driving performance was quantified by the $M$ and $S D$ of participants' speed, variation in lane position, the variation of the steering wheel position, as well as the number of steering wheel turns, regardless of their size. These measures were computed for the middle $850 \mathrm{~m}$ that the driver traveled to avoid the influence of merging onto or exiting the freeway. The Open DS software created a log file of the vehicle's lane position and speed. The files were imported into a custom Matlab application for the computation of the dependent variables.

Ten percent of the data were randomly remeasured for reliability. Across all dependent variables, the average correlation between the original and the remeasured data was .965 . The reliability data showed that the only difference during remeasurement could be attributed to the selection of the decibel (dB) floor, which may have slightly changed the $S D$ of intensity.

\section{Results}

Changes in the dependent measures across the single and dual-task conditions were tested with SPSS 23 software using a repeated measures analysis of variance (ANOVA). Within- 
subject factors included the task conditions (isolated versus concurrent), and between-subjects factors included group (age) and gender. Post hoc testing (Tukey HSD) was used to examine the significant differences between the groups in greater detail. All results presented below showed significant effects in the ANOVA testing at $p<.05$. The descriptive statistics for the speaking time ratio, average intensity, $S D$ of intensity, and $S D$ of $F_{0}$, are shown in Tables $1,2,3$, and 4, respectively. Because the $\mathrm{F}_{0}$ differs by about an octave by gender, the $\mathrm{F}_{0}$ values were converted into semitones to allow a direct comparison of variability for both men and women. The descriptive statistics for lane position variability, average speed, $S D$ of speed, $S D$ of steering wheel position, and number of steering wheel turns are presented in Tables, 5, 6, 7, 8, and 9, respectively.

\section{Table 1}

Descriptive Statistics for Average Speaking Time Ratio

\begin{tabular}{cccccc} 
& & \multicolumn{2}{c}{ Talking Only } & \multicolumn{2}{c}{ Driving and Talking } \\
\cline { 3 - 6 } Gender & Group & $M$ & $S D$ & $M$ & $S D$ \\
\hline \multirow{3}{*}{ Female } & 20 & 0.79 & 0.11 & 0.77 & 0.13 \\
& 40 & 0.74 & 0.07 & 0.74 & 0.07 \\
& 60 & 0.72 & 0.07 & 0.68 & 0.06 \\
\hline \multirow{3}{*}{ Male } & 20 & 0.72 & 0.07 & 0.73 & 0.07 \\
& 40 & 0.73 & 0.07 & 0.68 & 0.08 \\
& 60 & 0.75 & 0.08 & 0.69 & 0.07 \\
\hline
\end{tabular}


Table 2

Descriptive Statistics for Average Intensity (dB SPL at $50 \mathrm{~cm})$

\begin{tabular}{cccccc} 
& & \multicolumn{2}{c}{ Talking Only } & \multicolumn{2}{c}{ Driving and Talking } \\
\cline { 3 - 6 } Gender & Group & $M$ & $S D$ & $M$ & $S D$ \\
\hline \multirow{3}{*}{ Female } & 20 & 64.96 & 3.37 & 65.18 & 3.80 \\
& 40 & 65.26 & 4.17 & 65.88 & 3.79 \\
& 60 & 66.69 & 3.24 & 66.82 & 3.22 \\
\hline \multirow{3}{*}{ Male } & 20 & 64.30 & 5.09 & 64.86 & 4.89 \\
& 40 & 68.35 & 4.58 & 68.36 & 5.13 \\
& 60 & 67.31 & 3.90 & 67.71 & 4.50 \\
\hline
\end{tabular}

Table 3

Descriptive Statistics for Standard Deviation of Intensity in $\mathrm{dB}$

\begin{tabular}{cccccc} 
& & \multicolumn{2}{c}{ Talking Only } & \multicolumn{2}{c}{ Driving and Talking } \\
\cline { 3 - 6 } Gender & Group & $M$ & $S D$ & $M$ & $S D$ \\
\hline \multirow{3}{*}{ Female } & 20 & 5.72 & 0.88 & 5.51 & 0.81 \\
& 40 & 6.75 & 0.84 & 6.78 & 0.64 \\
& 60 & 6.68 & 0.83 & 6.86 & 0.79 \\
\hline \multirow{3}{*}{ Male } & 20 & 6.89 & 0.99 & 6.40 & 0.99 \\
& 40 & 7.28 & 0.43 & 7.17 & 0.69 \\
& 60 & 7.04 & 0.90 & 6.99 & 0.90 \\
\hline
\end{tabular}

Table 4

Descriptive Statistics for Standard Deviation of Fundamental Frequency in Semitones

\begin{tabular}{cccccc} 
& & \multicolumn{2}{c}{ Talking Only } & \multicolumn{2}{c}{ Driving and Talking } \\
\cline { 3 - 6 } Gender & Group & $M$ & $S D$ & $M$ & $S D$ \\
\hline \multirow{3}{*}{ Female } & 20 & 2.59 & 0.56 & 2.59 & 0.68 \\
& 40 & 2.58 & 0.66 & 2.72 & 0.56 \\
& 60 & 2.61 & 0.88 & 2.60 & 0.72 \\
\hline \multirow{3}{*}{ Male } & 20 & 2.04 & 0.62 & 2.14 & 0.71 \\
& 40 & 2.89 & 0.71 & 3.00 & 0.74 \\
& 60 & 2.50 & 0.59 & 2.47 & 0.38 \\
\hline
\end{tabular}


Table 5

Descriptive Statistics for Standard Deviation of Lane Position (Arbitrary Units)

\begin{tabular}{cccccc} 
& & \multicolumn{2}{c}{ Driving Only } & \multicolumn{2}{c}{ Driving and Talking } \\
\cline { 3 - 6 } Gender & Group & $M$ & $S D$ & $M$ & $S D$ \\
\hline \multirow{3}{*}{ Female } & 20 & 0.27 & 0.11 & 0.26 & 0.08 \\
& 40 & 0.30 & 0.10 & 0.51 & 0.29 \\
& 60 & 0.35 & 0.09 & 0.35 & 0.11 \\
\hline \multirow{3}{*}{ Male } & 20 & 0.25 & 0.08 & 0.20 & 0.06 \\
& 40 & 0.33 & 0.09 & 0.29 & 0.11 \\
& 60 & 0.44 & 0.22 & 0.38 & 0.19 \\
\hline
\end{tabular}

Table 6

Descriptive Statistics for Average Speed in $\mathrm{km} / \mathrm{h}$

\begin{tabular}{cccccc} 
& & \multicolumn{2}{c}{ Driving Only } & \multicolumn{2}{c}{ Driving and Talking } \\
\cline { 3 - 6 } Gender & Group & $M$ & $S D$ & $M$ & $S D$ \\
\hline \multirow{3}{*}{ Female } & 20 & 100.28 & 1.82 & 100.72 & 1.12 \\
& 40 & 99.45 & 1.79 & 99.70 & 2.96 \\
& 60 & 99.58 & 2.58 & 98.18 & 4.81 \\
\hline \multirow{3}{*}{ Male } & 20 & 99.33 & 1.57 & 100.26 & 3.35 \\
& 40 & 99.08 & 2.17 & 99.24 & 3.57 \\
& 60 & 99.13 & 2.77 & 98.33 & 3.85 \\
\hline
\end{tabular}

Table 7

Descriptive Statistics for Standard Deviation of Speed in $\mathrm{km} / \mathrm{h}$

\begin{tabular}{cccccc} 
& & \multicolumn{2}{c}{ Driving Only } & \multicolumn{2}{c}{ Driving and Talking } \\
\cline { 3 - 6 } Gender & Group & $M$ & $S D$ & $M$ & $S D$ \\
\hline \multirow{3}{*}{ Female } & 20 & 2.88 & 1.77 & 5.40 & 2.92 \\
& 40 & 4.72 & 1.64 & 4.85 & 1.90 \\
& 60 & 5.80 & 1.63 & 6.53 & 2.21 \\
\hline \multirow{3}{*}{ Male } & 20 & 3.20 & 1.40 & 4.31 & 2.25 \\
& 40 & 4.25 & 2.06 & 4.32 & 1.80 \\
& 60 & 6.34 & 3.13 & 7.06 & 2.68 \\
\hline
\end{tabular}


Table 8

Descriptive Statistics for Standard Deviation of Steering Wheel Position (Arbitrary Units)

\begin{tabular}{cccccc} 
& & \multicolumn{2}{c}{ Driving Only } & \multicolumn{2}{c}{ Driving and Talking } \\
\cline { 3 - 6 } Gender & Group & $M$ & $S D$ & $M$ & $S D$ \\
\hline \multirow{3}{*}{ Female } & 20 & 0.0013 & 0.0007 & 0.0018 & 0.0006 \\
& 40 & 0.0020 & 0.0008 & 0.0026 & 0.0012 \\
& 60 & 0.0021 & 0.0016 & 0.0037 & 0.0042 \\
\hline \multirow{3}{*}{ Male } & 20 & 0.0012 & 0.0006 & 0.0015 & 0.0007 \\
& 40 & 0.0014 & 0.0011 & 0.0020 & 0.0020 \\
& 60 & 0.0026 & 0.0013 & 0.0036 & 0.0024 \\
\hline
\end{tabular}

Table 9

Descriptive Statistics for Average Number of Steering Wheel Turns

\begin{tabular}{cccccc} 
& & \multicolumn{2}{c}{ Driving Only } & \multicolumn{2}{c}{ Driving and Talking } \\
\cline { 3 - 6 } Gender & Group & $M$ & \multicolumn{1}{c}{$S D$} & $M$ & $S D$ \\
\hline \multirow{3}{*}{ Female } & 20 & 26.30 & 10.54 & 42.30 & 16.69 \\
& 40 & 31.20 & 9.96 & 46.40 & 12.85 \\
& 60 & 29.40 & 15.56 & 46.60 & 26.16 \\
\hline \multirow{3}{*}{ Male } & 20 & 23.10 & 5.38 & 42.00 & 11.24 \\
& 40 & 21.10 & 8.16 & 33.90 & 10.56 \\
& 60 & 33.30 & 11.82 & 56.60 & 29.19 \\
\hline
\end{tabular}

\section{Divided Attention Effects}

Speech variables. The average speaking time ratio differed significantly between the isolated and the divided attention conditions, $F(1,54)=6.856 ; p=.011, \eta_{p}^{2}=.113$. As seen in Figure 1, the participants' speaking time ratio decreased in the divided attention condition compared to the talking only condition across all ages and for both genders. This decrease was greater in the 60 s group, although the difference did not reach significance as a condition by group interaction. 


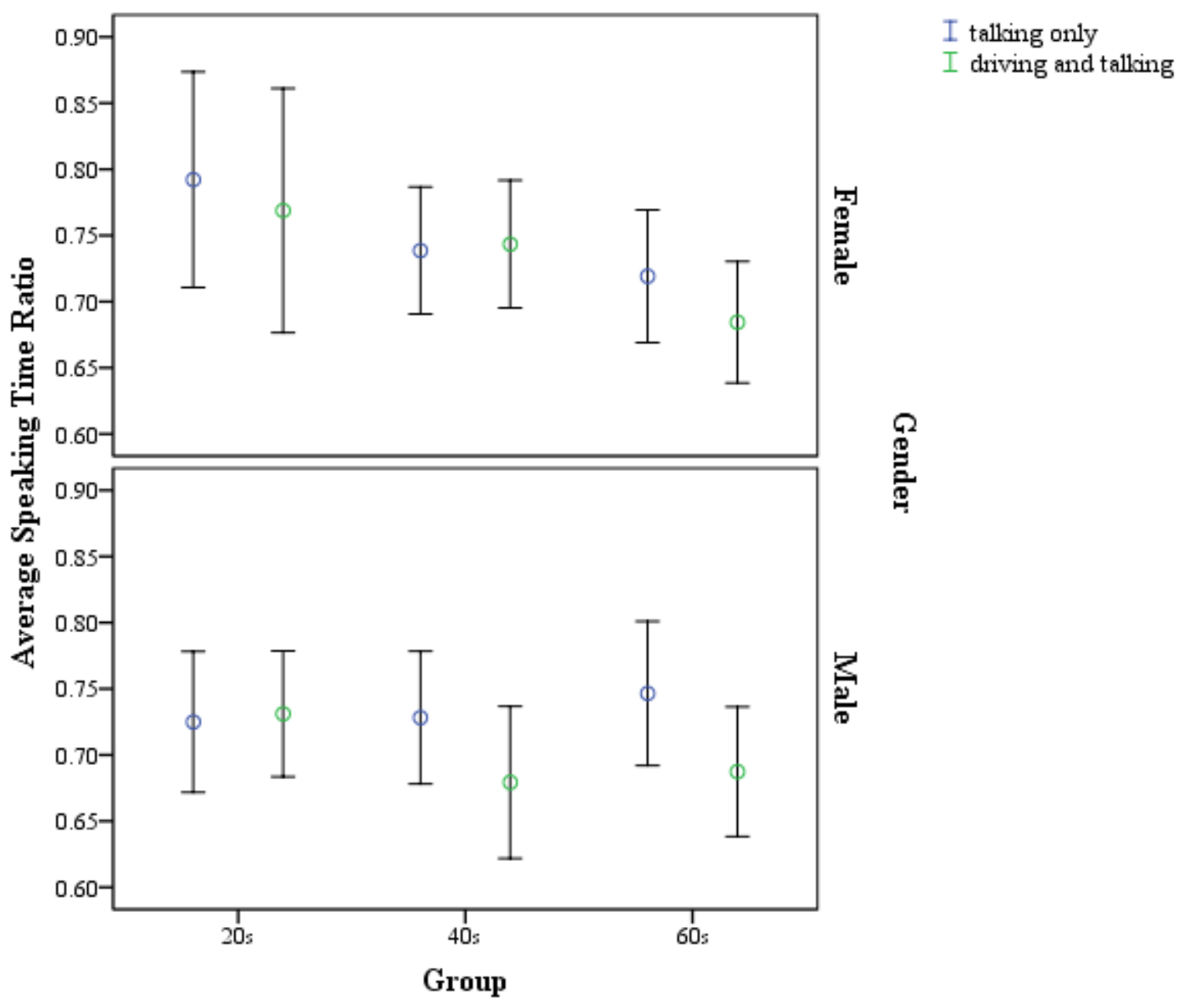

Figure 1. Mean (and 95\% confidence interval) of the speaking time ratio.

The $M$ intensity also differed significantly between the conditions, $F(1,54)=5.213 ; p=$ $.026, \eta_{p}^{2}=.088$. As seen in Figure 2 , the $M$ intensity increased in the divided attention condition. 


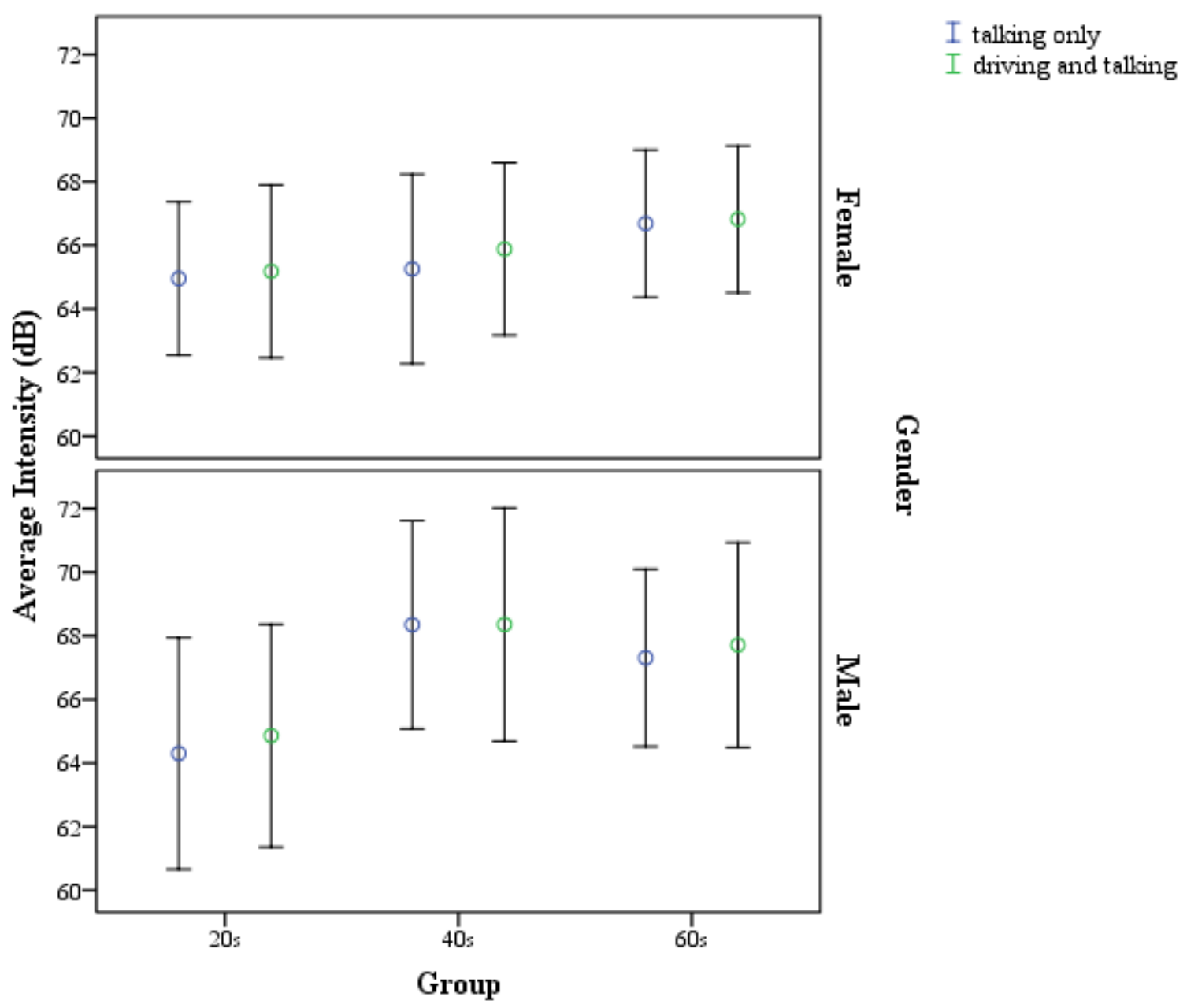

Figure 2. Mean (and 95\% confidence interval) intensity (dB SPL at $50 \mathrm{~cm}$ ).

Driving variables. The average speed slightly increased for the $20 \mathrm{~s}$ and 40 s groups and slightly decreased for the 60s group during the divided attention condition. ANOVA testing revealed, however, that these results did not reach statistical significance. The $S D$ of speed differed significantly between the two conditions, $F(1,54)=7.710 ; p=.008, \eta_{p}^{2}=.125$. As shown in Figure 3, the participants' variability in speed increased during the divided attention condition compared to the isolated driving task. 


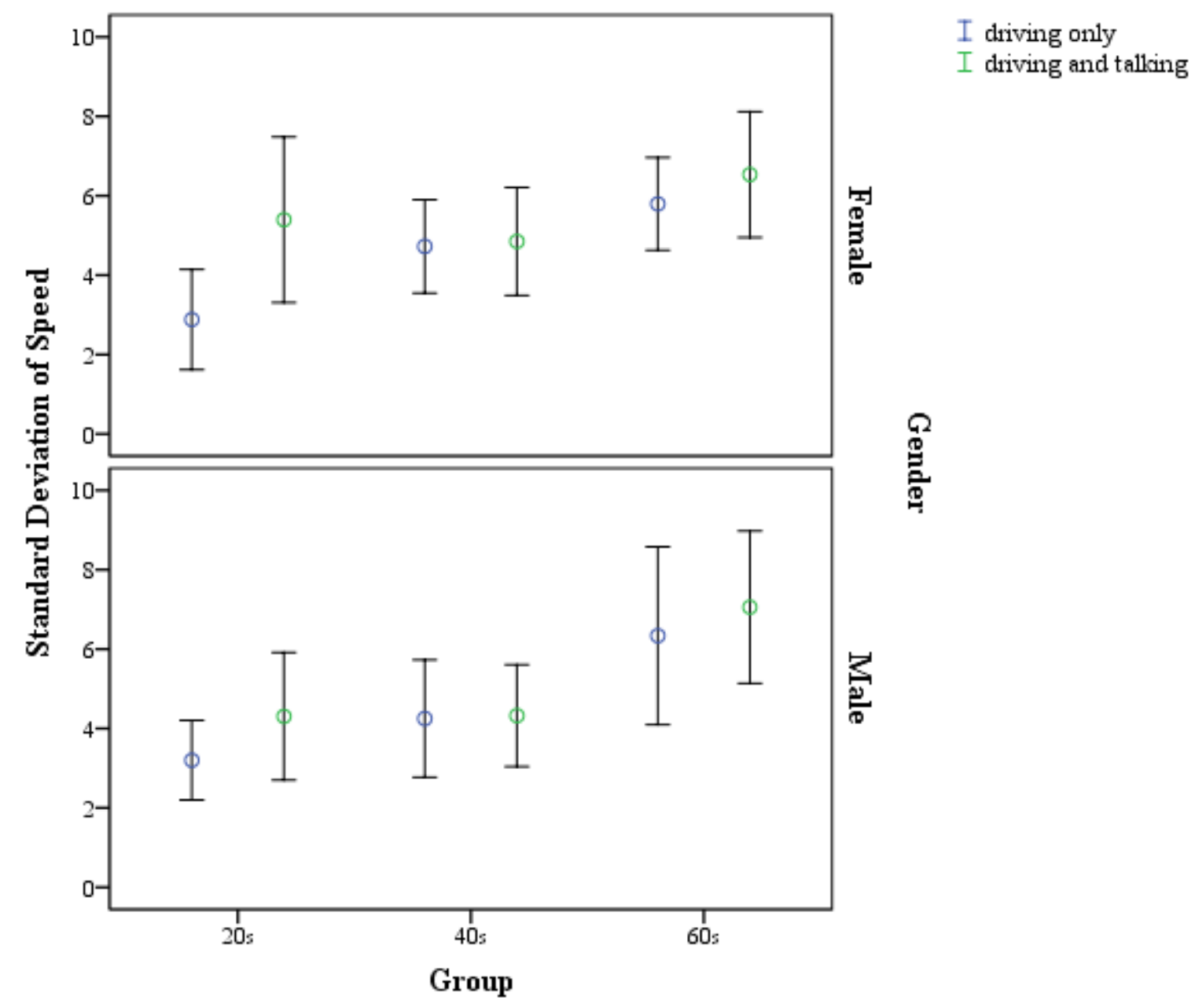

Figure 3. Mean (and 95\% confidence interval) of the standard deviation of speed in $\mathrm{km} / \mathrm{h}$.

The $S D$ of steering wheel position also differed significantly between the two conditions, $F(1,54)=11.157 ; p=.002, \eta_{p}^{2}=.171$. As shown in Figure 4 , the position of the steering wheel varied more in the divided attention condition compared to the driving only condition. 


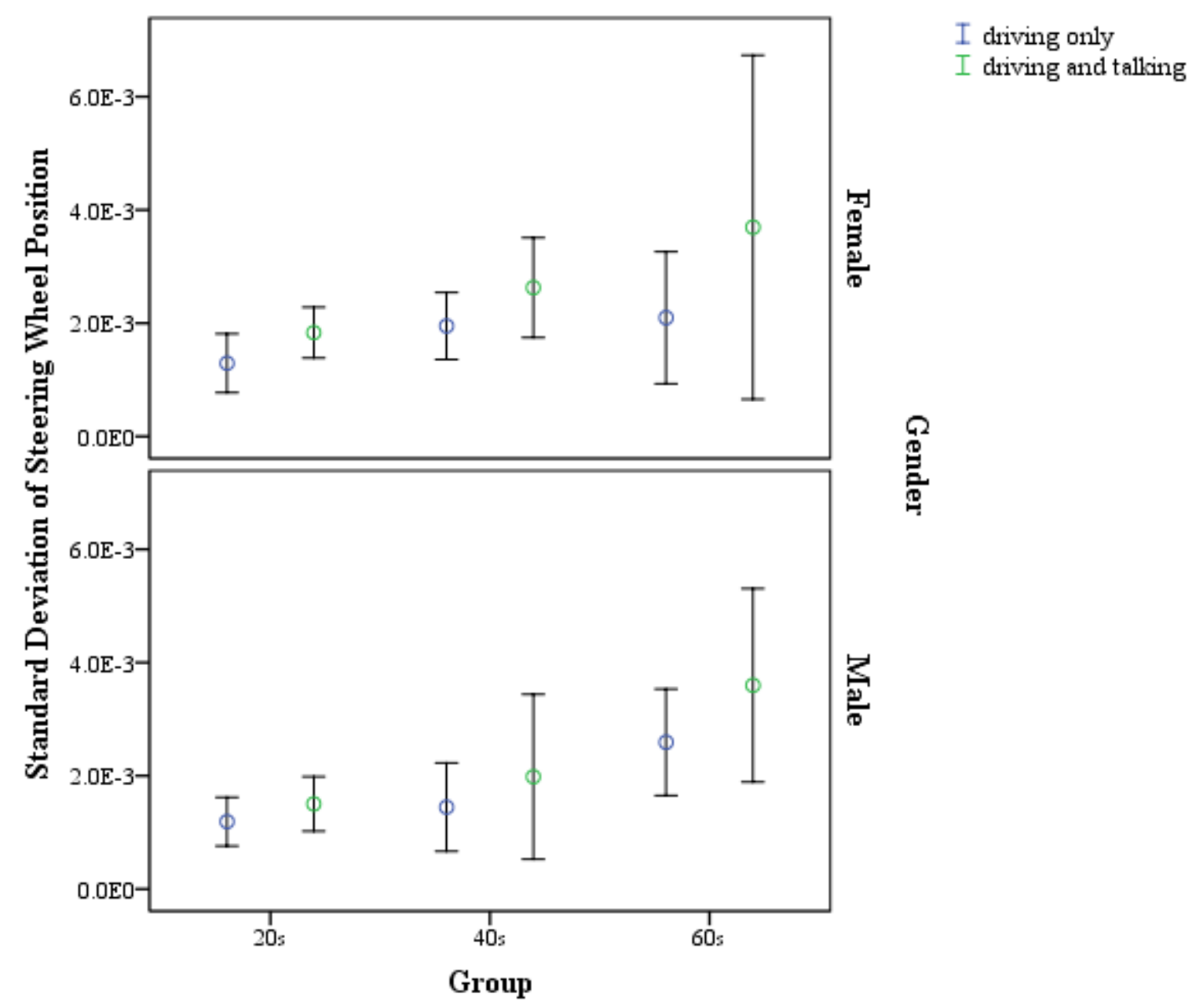

Figure 4. Mean (and 95\% confidence interval) of standard deviation of steering wheel position (arbitrary units).

The number of steering wheel turns or corrections that a participant made also differed significantly between conditions, $F(1,54)=98.633 ; p<.001, \eta_{p}^{2}=.646$. As shown in Figure 5, the average number of steering wheel adjustments increased in the divided attention condition. 


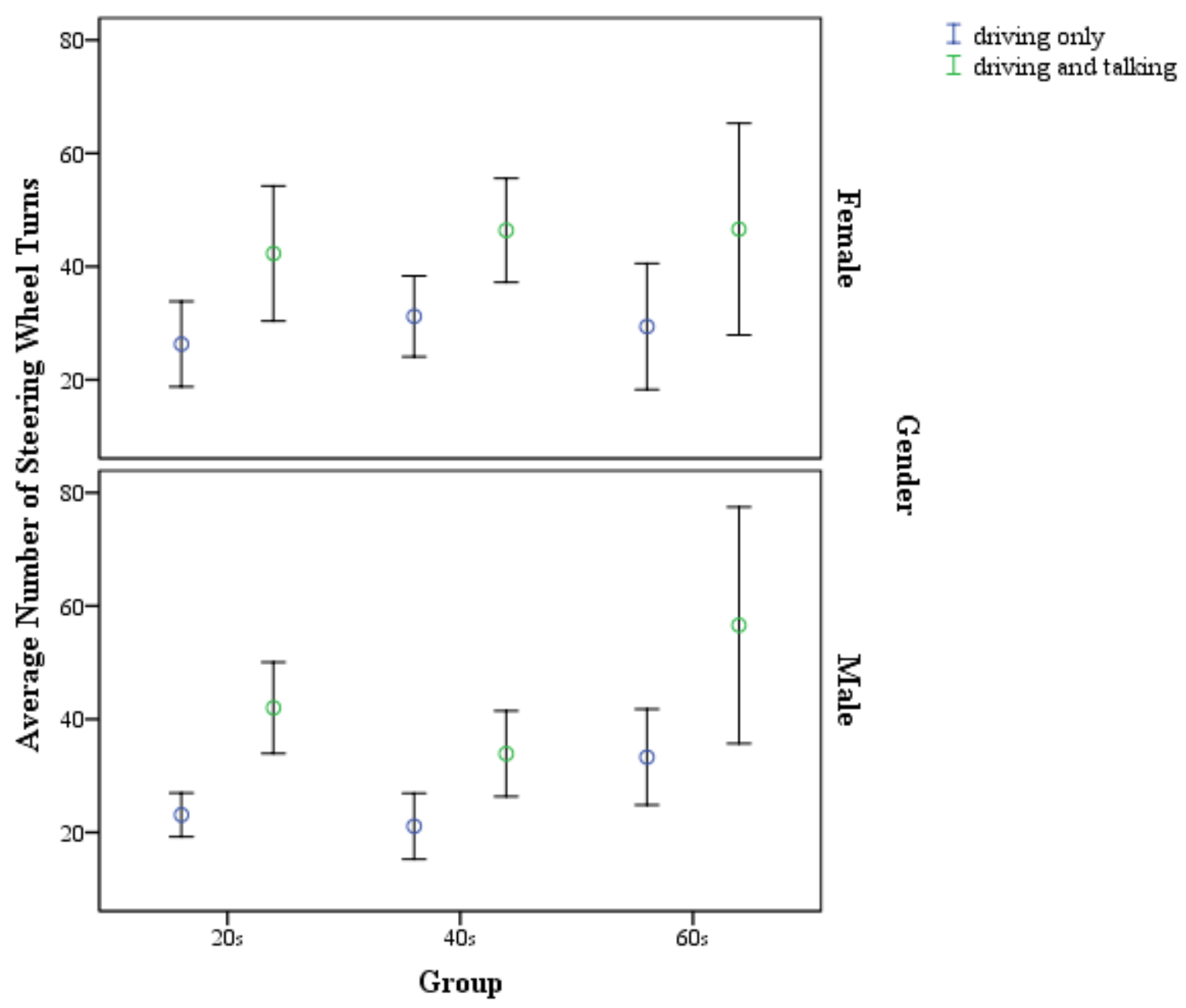

Figure 5. Mean (and 95\% confidence interval) number of steering wheel turns.

\section{Age Effects}

Speech variables. There was a significance between subjects effect for the $S D$ of intensity across the age groups, $F(1,54)=8.728 ; p=.001, \eta_{p}^{2}=.153$. Figure 6 shows that the 20s group had less variation in their intensity compared to the other age groups. Post hoc testing showed that the 20 s group differed significantly compared to the 40 s group, $p=.001$ and the 60 s group, $p=.004$. 


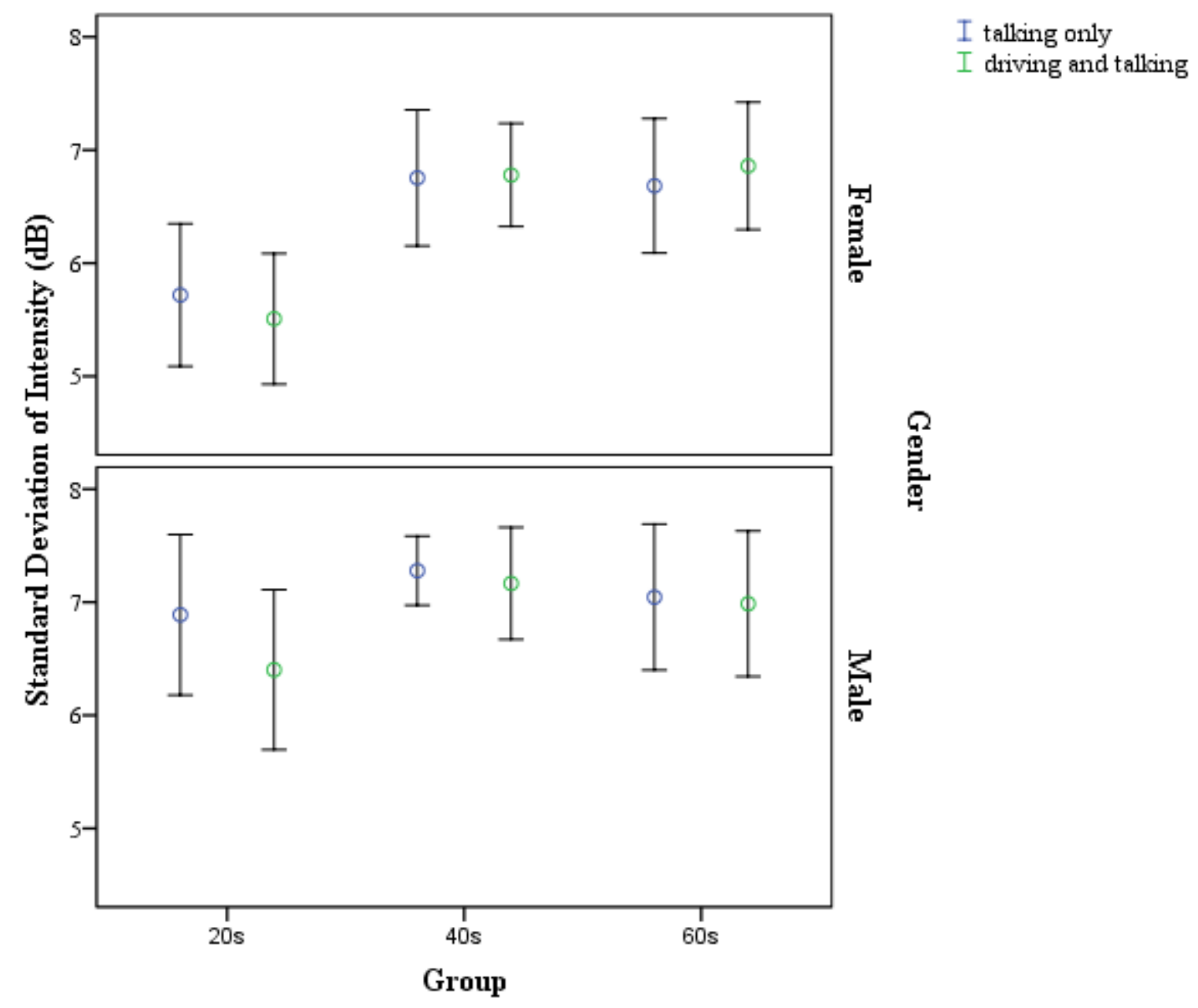

Figure 6. Mean (and 95\% confidence interval) standard deviation of intensity (dB).

The $S D$ of $\mathrm{F}_{0}$ in semitones differed across the age groups, $F(2,54)=2.919 ; p=.063, \eta_{p}^{2}$

$=.098$. Although this between subjects effect was not statistically significant at $p<.05$, post hoc testing revealed that the 20 s group differed significantly from the 40 s group, $p=.050$. As shown in Figure 7, the variation in $\mathrm{F}_{0}$ was lower for the 20s group compared to the 40s group, especially in men. 


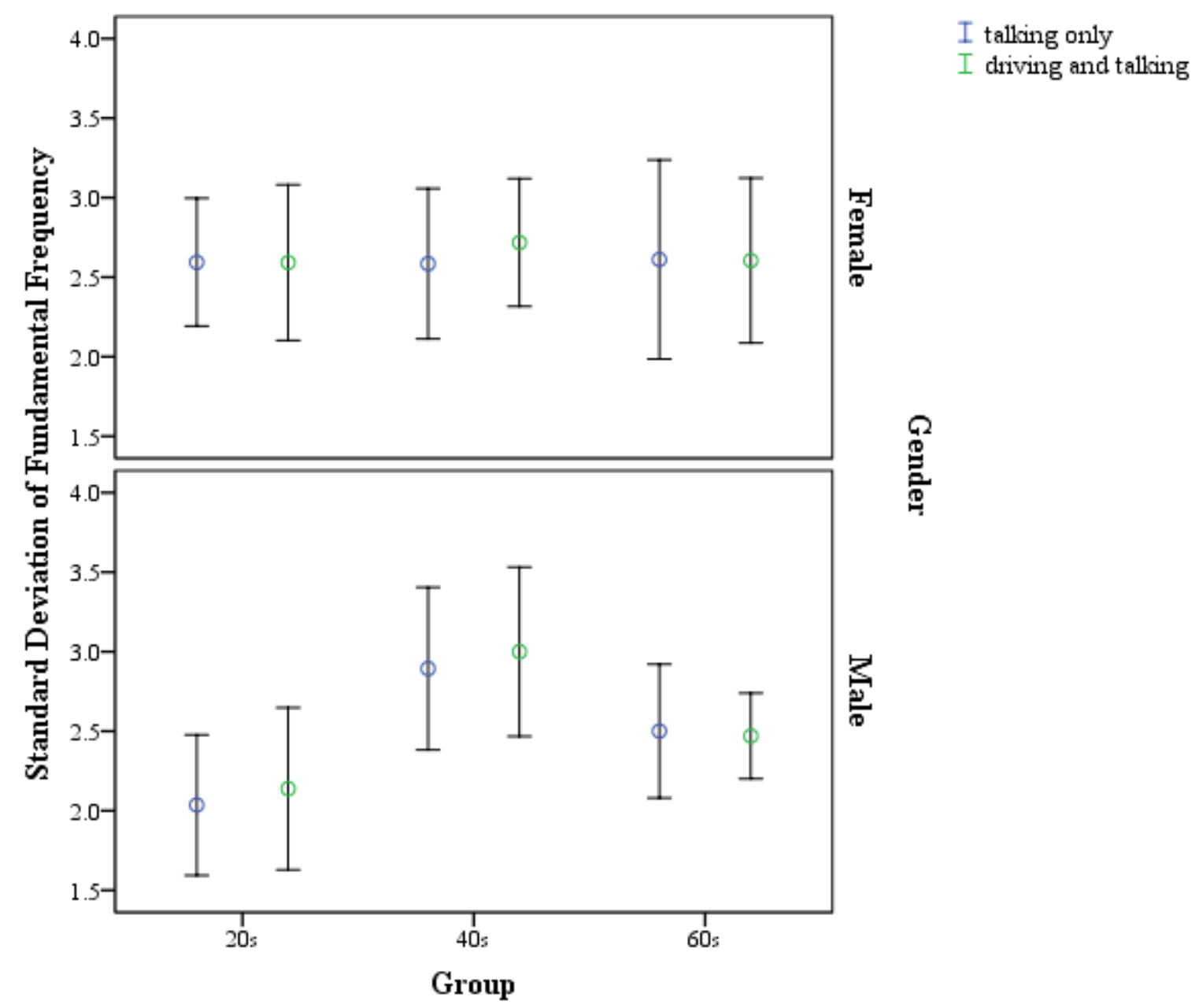

Figure 7. Mean (and 95\% confidence interval) standard deviation of fundamental frequency in semitones.

Driving variables. The ANOVA revealed a significant condition by group interaction for lane position deviation, $F(2,54)=3.672 ; p=.032, \eta_{p}^{2}=.120$. As seen in Figure 8, there was a slight decrease in lane deviation during the divided attention condition, with the exception of the females in the 40s group, who had more variability in their lane position during the divided attention condition compared to the other participants. The figure also shows that the 20 s group deviated from the center of the lane less than the other age groups in both conditions. Betweensubject testing also revealed a significant difference for age group, $F(2,54)=7.674 ; p=.001$, 
$\eta_{p}^{2}=.221$. Post hoc testing revealed that the 20 s group had less lane position variability than the 40s group, $p=.009$ and the 60s group, $p=.002$.

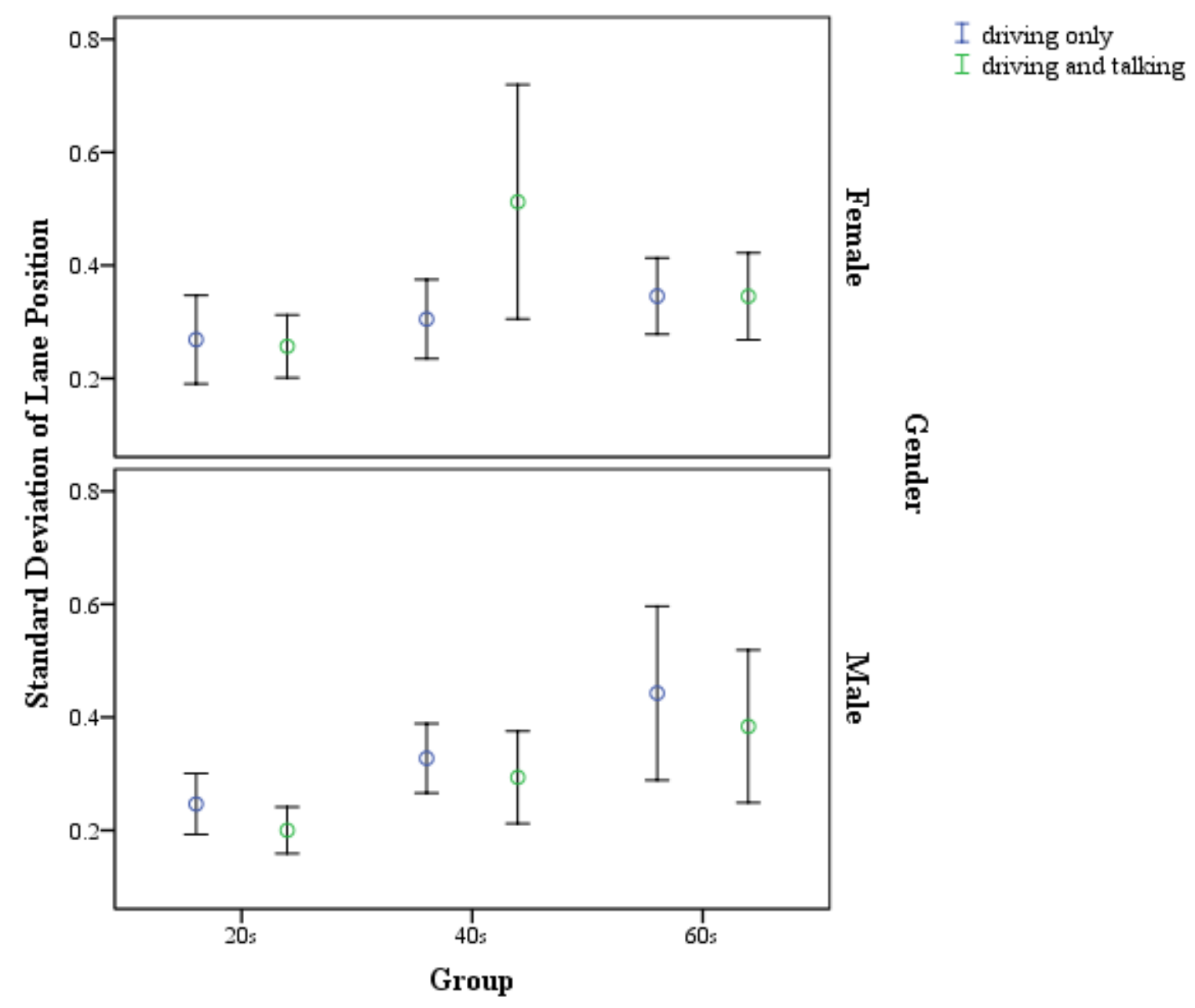

Figure 8. Mean (and 95\% confidence interval) standard deviation of lane position (arbitrary units).

Between-subjects testing revealed that the $S D$ of speed was significantly different by group, $F(2,54)=10.378 ; p<.001, \eta_{p}^{2}=.278$. As seen in Figure 3 above, there was an increase in speed variation during the divided attention condition across all ages, but the 60s group had more speed variation in both conditions than the other two groups. Post hoc testing revealed that the 60s group differed significantly from the 20s group, $p<.001$ and the 40 s group, $p=.004$. 
Between-subjects testing revealed the $S D$ of steering wheel position to be significantly different across the groups, $F(2,54)=5.303 ; p=.008, \eta_{p}^{2}=.164$. As seen in Figure 4 above, there was an increase in steering wheel position variation during the divided attention condition across all ages, but the 20s group had less variation in both conditions than the other two groups, and the 60s group had the most variation in both conditions. Post hoc testing showed that the 20s group differed significantly from the 60 s group, $p=.006$.

\section{Gender Effects}

Speech variables. $S D$ of intensity was found to be the only speech variable affected by gender. Between-subject testing revealed that the variation of intensity was significantly different by gender, $F(1,54)=5.303 ; p=.008, \eta_{p}^{2}=.164$. As seen in Figure 6 earlier, males across all age groups had more variation in their intensity level compared to females.

Driving variables. Within-subject testing revealed a significant condition by gender interaction for lane position deviation, $F(1,54)=3.672 ; p=.008, \eta_{p}^{2}=.123$. As stated earlier and shown in Figure 7, the females in the 40s group deviated further away from the center of the lane compared to the males across all age groups. This was the only driving variable found to be affected by gender.

\section{Discussion}

This study was designed to investigate the anticipated bidirectional interference between speaking and a driving task. It was hypothesized that speaking and driving performance would be negatively impacted when performed simultaneously. It was also designed to examine the effects of age on divided attention performance, with the hypothesis that younger adults would experience less dual-task interference than older adults. The results provide evidence that dual tasking negatively influenced performance on both tasks. 


\section{Effects of Driving on Speech Performance}

As predicted in our hypothesis, the divided attention condition yielded lower speaking time ratios than the isolated talking condition for all groups, meaning that they had more pauses in their speech when they were driving at the same time. This could be a result of the limited attentional resources available for the individual to process what to say next due to the cognitive demands of the driving task, and thus supports limited capacity theories of divided attention (Pashler \& Johnston, 1998). The increased pausing in the dual-task condition could also be explained by Wickens' time-sharing model: that the alternation of attention between the two tasks was not fast enough, and thus performance in the speech task declined (Wickens, 1981).

Because previous studies have focused on how talking impacts driving performance, there are few reports to compare with the present results. Oomen and Postma (2001) found comparable results in their study, where participants performed a storytelling task and a blind tactile-form recognition task simultaneously; the number of filled pauses and disfluencies increased for the dual-task condition. Although the tasks differed from the current study, the divided attention conditions from both studies resulted in an increased number of pauses in speech.

Contrary to our predictions, the divided attention condition resulted in an increase in the average intensity of speech compared to the isolated condition. In other words, across all ages and for both genders, participants were louder when they were driving. Similar findings were reported in previous studies of concurrent speech and manual motor tasks (Dromey \& Shim, 2008; Dromey \& Bates, 2005). It could be speculated that participants felt the need to talk more loudly to convey their message, since the driving task also required their attention. 
Speaking while concurrently driving caused subtle changes in the way participants talked. Some studies have shown how dual-task demands impact speech comprehension, story retelling, and memory, but reports of the effects of driving on the way speech is produced have been very limited (Becic et al., 2010; Cao \& Liu, 2013). However, the changes in speech are consistent with previous literature on the interference between speech and nonspeech tasks; dual-task conditions caused a decline in speech performance (Bailey \& Dromey, 2015; Dromey \& Shim, 2008; Dromey \& Bates, 2005). Although these studies relied on repetitive speaking tasks and speech kinematic measures, the general trends of dual-task performance are similar to the current study.

Modulation of intensity and $\mathrm{F}_{0}$ creates intonation patterns in natural speech to convey meaning and emotion. Reduced variability in intensity and $\mathrm{F}_{0}$ can result in less natural speech. Between subject tests revealed that the $S D$ of intensity differed significantly by group and gender. Contrary to our hypothesis, post hoc results revealed significant differences between the 20 s group and the other groups; the 20s group had less variation in intensity across both conditions compared to other age groups. It was also found that the men had greater intensity variability across both conditions compared to the women.

Post hoc testing showed a significant difference in the $S D$ of $\mathrm{F}_{0}$ between the 20s group and the 40s group. The 20s group had less variation in $\mathrm{F}_{0}$ across both conditions compared to the 40s group, meaning that the speech of the younger age group was more monopitch than the older groups. As shown in Figure 7, these trends may be attributable to the males in the 20s group because they had less variation in $\mathrm{F}_{0}$ compared to the other groups as well as the females.

The intonation patterns of the 20s group were unexpected because it was predicted that the older-aged group would have less variation in intensity and $\mathrm{F}_{0}$ than the younger groups. The 
lower variability of both intensity and $\mathrm{F}_{0}$ in the 20 s group may be because younger speakers rely less on intonation patterns to convey meaning in their speech and more on the actual words or language they use, although this is speculative. These findings warrant further investigation through the examination of the intonation patterns of younger and older adults in future studies.

The current study showed that males had more variation in intensity compared to the females, but that the females had more variation in $\mathrm{F}_{0}$ compared to the males. These trends, however, were only found in the 20s group. An explanation for these findings could be that to convey meaning in speech, males tend to rely more on variation in loudness, whereas females rely more on variation in $\mathrm{F}_{0}$. These results suggest that further investigation of $\mathrm{F}_{0}$ and intensity patterns among genders and different ages is needed.

\section{Effects of Speaking on Driving Performance}

As predicted, the $S D$ of speed for all participants increased in the dual-task condition, meaning that the participants had more variation in their speed when they were speaking about their selected topics. These results differ from those reported in some previous studies (Becic et al., 2010). The dual-task condition also resulted in a slight increase in average speed for the two younger groups, and a slight decrease for the 60 s group, although these results were not statistically significant. These trends support the findings from previous studies reporting that participants drove faster when performing speech production and comprehension tasks, conversing on a phone, or participating in an emotional conversation (Dula et al., 2011; Beede \& Kass, 2006; Kubose et al., 2006). Dula et al., however, found that there was no difference in speed maintenance between a mundane conversation condition and the isolated driving task. These findings from the literature may explain the increased speed variation in the current study. Similar to the varying levels of emotion in conversations in Dula et al.'s study, the varying levels 
of cognitive workload of the topics may have caused more attention to be occupied with the speaking task instead of the driving task, and thus the $S D$ and average speed increased.

This effect may also be explained by the limited capacity theories, or possibly by structural theories such as the bottleneck effect, which suggests that cognitive processes are carried out in a sequential order. Thus, in dual-task conditions, a bottleneck occurs as the attentional processes are focused on completing one task before attending to the second task, causing the performance in the second task to become delayed (Pashler \& Johnston, 1998; Wickens, 1981). If the drivers' attention was focused on their response to the topic, there could have been a delay in monitoring and maintaining their speed until they processed what they wanted to say. This delay in attentional processing could explain the increased variation of speed in the divided attention condition.

The $S D$ of steering wheel position, as well as the number of steering wheel turns, both increased in the divided attention condition. These results differ from those in a previous study, which reported that steering wheel control was not affected during dual-task conditions (Cao \& Liu, 2013). However, these results may be attributable to the sensitivity of the steering wheel in the present experiment. Thus, when the participants' attention was divided between the two tasks, even a slight decrease in attention to the steering wheel control may have caused a decline in steering wheel stability. Despite this decline in performance, the $S D$ of lane position decreased for five of the six groups in the divided attention condition, meaning that lane position was less variable when participants were also talking. Although contrary to our hypothesis, these results provide support for previous findings (Becic et al., 2010; Beede \& Kass, 2006; Kubose et al., 2006). These findings also provide support for the hierarchal control theory as the process of lane maintenance is considered to be dependent on the inner loop of attentional processing 
(Medeiros-Ward et al., 2014). In other words, the more attention that is given to lane maintenance, the worse the performance. Thus based on this theory, the divided attention condition resulted in better lane maintenance as less attention was given to the task.

The $S D$ of lane position did not change in the dual-task condition as a significant main effect in the ANOVA, but it was found to interact significantly with age and gender because of the 40 s female group. This is because 4 of the 10 women merged late onto the freeway in the concurrent speaking condition. As a consequence, they were still merging when the lane position measurements had started. Although these errors skewed the data, the same participants did not merge late in the driving only condition. Thus, the distracting nature of the divided attention condition may have caused the women to merge late and increase their $S D$ of lane position. Post hoc testing also revealed that the 20s group was less variable in lane position than the older individuals. This could be because the younger group may have had more exposure to video games and simulators compared to the older groups.

The $S D$ of speed changed significantly as a main effect. Between subjects tests also revealed that the 60 s group differed significantly compared to the other groups because they had greater variability in their speed. This result could be attributed to the effects of the aging brain as divided attention performance declines in older populations (McDowd \& Craik, 1988). Strayer and Drews (2004), however, found that age was not a significant factor in driving performance when concurrently talking on a hands-free cell phone. The authors found different driving behaviors between the elderly and young-adults (e.g., following distance from the lead car, braking response time, etc.), but did not find any significant differences in overall driving performance. 
The $S D$ of steering wheel position increased for the divided attention condition for all ages, but the 20s group had the least amount of variation in both conditions and the 60 s group had the most. Post hoc testing showed this difference to be statistically significant. As suggested previously, this is likely because the 20s group may have more experience with video games compared to the 60 s group.

Although the current study provided some significant findings regarding age and gender effects in dual-task driving performance, it could be speculated that many of the findings might be attributed to the overly sensitive simulator hardware and the amount of exposure to video games and other simulations. Gender was found to have no significant effect on any driving measures, with the exception of the 40 s female group that merged late. Age was found to be a significant factor with regard to speed, lane position, and steering wheel control whereby the older participants showed poorer performance in these areas. These phenomena could be investigated further in future studies.

\section{Limitations of the Current Study and Directions for Future Research}

Most of the limitations in this study were related to the driving simulator setup, including the OpenDS software and the consumer-grade hardware. The software version that was used in this study offered few tasks that could provide quantifiable driving data. The Motorway task that was used only measured driving performance of the middle $850 \mathrm{~m}$ of the straight line course. The course did not involve any turns, stops, or other driving scenarios that may have required more attention. Although the driving task did require some attention, future studies could explore more complex driving tasks to increase the level of attention required of the participants.

The steering wheel was very sensitive when participants made turns, which may have contributed to the motion sickness reported by two of the females in the 60s group, as well as 
dizziness reported by two other females in the 60 s group. In fact, two additional females in the 60s group (not included in the ten female participants in the 60s group) had to withdraw from participation due to feelings of motion sickness. Future studies could explore programming the steering to be less sensitive, or using a better equipped simulator to eliminate these effects and to make the driving task more realistic.

In addition to the driving task limitations, the same monologue topics were not used for each participant for the speech task. Although the participants selected the topics to talk about beforehand, some topics were more relatable and easy to talk about than others. Thus, the topics that were more difficult to talk about may have required more processing or attention, which may have resulted in more pauses, decreased intensity or variation of intensity, or decreased $\mathrm{F}_{0}$ variation.

\section{Conclusion}

Few studies have considered the effects of divided attention conditions on acoustic measures of speech performance, especially in dual-task driving studies. The data not only confirm findings of previous studies of divided attention, but also provide insight into the effect of age in these conditions. The findings of the current study suggest that the way speech is produced is negatively impacted in dual-task driving conditions. The acoustic variables of speech that were adversely affected by the driving task were the speaking time ratio and the average level of intensity. The current findings also suggested that age and gender play a role in patterns of intensity and $\mathrm{F}_{0}$, although further research in this area is warranted. Several driving variables were affected by talking, including the $S D$ of speed, lane position, and steering wheel position, as well as the average speed and number of steering wheel turns. Age was a significant 
factor in the variation of speed and steering wheel position, although better equipment used in future studies may prove otherwise.

These findings have clinical implications for the treatment of patients with communication disorders. The setting and format of therapy sessions typically eliminates distractions. Although this may provide patients with an optimal environment to learn and practice their communication goals, it may limit the extent to which their skills will generalize as talking in the real world includes distractions. The findings of the current study thus suggest that divided attention conditions could be incorporated into therapy to help patients become more robust in their everyday communication. 


\section{References}

Bailey, D. J., \& Dromey, C. (2015). Bidirectional interference between speech and nonspeech tasks in younger, middle-aged, and older adults. Journal of Speech, Language, and Hearing Research, 58(6), 1637-1653. doi:10.1044/2015_JSLHR-S-14-0083

Ballard, K. J., Robin, D. A., Woodworth, G., \& Zimba, L. D. (2001). Age-related changes in motor control during articulator visuomotor tracking. Journal of Speech, Language, and Hearing Research, 44(4), 763-777. doi:10.1044/1092-4388(2001/060)

Becic, E., Dell, G. S., Bock, K., Garnsey, S. M., Kubose, T., \& Kramer, A. F. (2010). Driving impairs talking. Psychonomic Bulletin \& Review, 17(1), 15-21. doi:10.3758/PBR.17.1.15

Beede, K. E., \& Kass, S. J. (2006). Engrossed in conversation: The impact of cell phones on simulated driving performance. Accident Analysis \& Prevention, 38(2), 415-421. doi:10.1016/j.aap.2005.10.015

Bergen, B., Medeiros-Ward, N., Wheeler, K., Drews, F. A., \& Strayer, D. L. (2013). The crosstalk hypothesis: Why language interferes with driving. Journal of Experimental Psychology: General, 142(1), 119-130. doi:10.1037/a0028428

Boersma, P., \& Weenink, D. (2014). Praat (Version 5.4).

Cao, S., \& Liu, Y. (2013). Concurrent processing of vehicle lane keeping and speech comprehension tasks. Accident Analysis \& Prevention, 59, 46-54. doi:10.1016/j.aap.2013.04.038

Drews, F. A., Pasupathi, M., \& Strayer, D. L. (2008). Passenger and cell phone conversations in simulated driving. Journal of Experimental Psychology: Applied, 14(4), 392-400. doi:10.1037/a0013119 
Dromey, C., \& Bates, E. (2005). Speech interactions with linguistic, cognitive, and visuomotor tasks. Journal of Speech, Language, and Hearing Research, 48(2), 295-305. doi:10.1044/1092-4388(2005/020)

Dromey, C., \& Shim, E. (2008). The effects of divided attention on speech motor, verbal fluency, and manual task performance. Journal of Speech, Language, and Hearing Research, 51(5), 1171-1182. doi:10.1044/1092-4388(2008/06-0221)

Dula, C. S., Martin, B. A., Fox, R. T., \& Leonard, R. L. (2011). Differing types of cellular phone conversations and dangerous driving. Accident Analysis \& Prevention, 43(1), 187-193. doi:10.1016/j.aap.2010.08.008

Kubose, T., Bock, K., Dell, G., Garnsey, S., Kramer, A., \& Mayhugh, J. (2006). The effects of speech production and speech comprehension on simulated driving performance. Applied Cognitive Psychology, 20, 43-64. doi:10.1002/acp.1164

McDowd, J. M., \& Craik, F. I. (1988). Effects of aging and task difficulty on divided attention performance. Journal of Experimental Psychology: Human Perception Performance, 14(2), 267-280.

Medeiros-Ward, N., Cooper, J. M., \& Strayer, D. L. (2014). Hierarchical control and driving. Journal of Experimental Psychology: General, 143(3), 953-958. doi:10.1037/a0035097

Oomen, C. C., \& Postma, A. (2001). Effects of divided attention on the production of filled pauses and repetitions. Journal of Speech, Language, and Hearing Research, 44(5), 9971004. doi:10.1044/1092-4388(2001/078)

Pashler, H. \& Johnston, J. (1998). Attentional Limitations in Dual-Task Performance. In H. Pashler (Ed.), Attention (pp. 155-189). Hove, UK: Psychology Press. 
Strayer, D. L., Cooper, J. M., Turrill, J., Coleman, J., Medeiros-Ward, N., \& Biondi, F. (2013). Measuring cognitive distraction in the automobile. Retrieved from AAA Foundation for Traffic Safety: https://www.aaafoundation.org/sites/default/files/MeasuringCognitiveDistractions.pdf

Strayer, D. L., \& Drews, F. A. (2004). Profiles in driver distraction: Effects of cell phone conversations on younger and older drivers. Human Factors, 46(4), 640-649.

Strayer, D. L. \& Drews, F. A. (2007). Cell-phone-induced driver distraction. Current Directions in Psychological Science, 16(3), 128-131. doi:10.111/j.1467-8721.2007.00489.x

Strayer, D. L. \& Johnston, W. A. (2001). Driven to distraction: Dual-task studies of simulated driving and conversing on a cellular telephone. Psychological Science, 12(6), 462-466. doi:10.1111/1467-9280.00386

Strayer, D. L., Watson, J. M., \& Drews, F. A. (2010). Cognitive distraction while multitasking in the automobile. In B. Ross (Ed.), The psychology of learning and motivation (Vol. 54, pp. 29-58). Burlington, VA: Elsevier Inc.

Wickens, C. D. (1981). Processing resources in attention, dual task performance, and workload assessment (No. EPL-81-3/0NR-81-3). Champaign, IL: University of Illinois at UrbanaChampaign. 


\section{APPENDIX A: Annotated Bibliography}

Bailey, D., \& Dromey, C. (2015). Bidirectional interference between speech and nonspeech tasks in younger, middle-aged, and older adults. Journal of Speech, Language, and Hearing Research, 58(6), 1637-1653. doi:10.1044/2015_JSLHR-S-14-0083

Objective: The purpose of the study was to examine the effects of aging and concurrently performed speech and nonspeech tasks. Method: Participants in the study included 20 young adults $(M=22.95$ years of age), 20 middle-aged adults $(M=45.60$ years of age), and 20 older adults $(M=63.20$ years of age). Participants completed a 30 -second practice trial of the four tasks prior to the experimental trials to help them become familiar with the task. One of the tasks was a speech task, in which participants were instructed to repeat the sentence I saw Patrick pull a wagon packed with apples every time they heard a tone. This task was completed under a single-task condition first, then combined with three other nonspeech tasks (linguistic, cognitive, and manual motor tasks) to create dual-task conditions. Speech task measures were based on duration, spatiotemporal index (STI) for the lower lip, and SPL. The following measurements were also taken from the word packed: lower lip displacement and peak velocity, and upper lip and lower lip correlation. The nonspeech tasks were also completed individually before the combined conditions. The linguistic task involved a semantic decision activity. Participants would be presented with two words, a noun and a verb, on a screen. The participants decided whether the two words presented were semantically related or not, and were given 60 seconds to complete as many pairs of words as possible. The cognitive task required participants to determine whether two fraction numbers presented on a screen were equal or not. The participants were given 60 seconds to complete as many numerical comparisons as they could. The linguistic and cognitive tasks were scored based on the total number of responses, the number of correct responses, and total percentage of accuracy in the task. The Purdue Pegboard Test was the manual motor task, which had participants put as many pegs as possible in the pegboard with both hands within 60 seconds. Scores for this task were based on the number of pegs placed within the given time frame. The scores from each of the isolated conditions were compared to the scores in the combined conditions. Results: The researchers found condition to have a significant main effect on speech task performance, as the performance in the isolated task conditions differed from the performance in the dual-task condition. The researchers found that the dual-task conditions led to the following effects in task performance: duration of the sentence was significantly longer during the linguistic and cognitive combined tasks compared to the speech-only task, lower lip displacement was reduced during the combined manual motor task compared to the speech-only task, upper lip and lower lip correlation became significantly more negative during the combined linguistic and cognitive tasks, STI of the lower lip increased in the combined conditions, and SPL increased significantly in the manual motor condition. Condition was also found to have a significant main effect on nonspeech task performance, as the number of correct and total responses significantly decreased in the combined linguistic condition compared to its isolated counterpart, as well as the number of correct responses significantly decreased in the combined cognitive condition compared to the isolated condition. Age was also found to have a significant main effect on segment duration of the speech task and all variables of the nonspeech tasks. The older age group had longer utterance duration during the speech task and decreased performance on all variables in the three nonspeech tasks. Conclusion: Based on these findings, the researchers concluded that task type and age affect 
speech performance during divided attention situations, and that these two factors interact with each other. The researchers found that the older age group had more difficulties in dual-task performance than the younger groups, which supports the findings of previous studies. Relevance to the current work: The study provides insight as to how age impacts dual-task performance and the effects on speech movements in divided attention situations. A limitation in this study is that the speech task lacked ecological validity, which is a factor that is being addressed in the current study.

Ballard, K. J., Robin, D. A., Woodworth, G., \& Zimba, L. D. (2001). Age-related changes in motor control during articulator visuomotor tracking. Journal of Speech, Language, and Hearing Research, 44(4), 763-777. doi:10.1044/1092-4388(2001/060)

Objective: This study was designed to investigate differences in articulatory motor control across different ages by measuring the lower lip, jaw, and laryngeal movements during a tracking task. Method: Participants in this study included 52 females and 35 males, all of whom were between 8:2 and 84:3 years old, had no history of speech, language, or neurological disorders, and had normal vision and hearing. Participants were positioned in front of a screen and were instructed to track a target signal, represented as a thin horizontal bar, using the specified articulator. The signals from the articulators' movements were transduced and represented as a dot for participants to track their signal in comparison to the target signal. The target signal moved in four conditions: three predictable conditions, and one unpredictable condition. The researchers used a strain gauge cantilever system to measure the participants' lip and jaw movements, as well as used microphones to measure fundamental frequency, representing laryngeal movements. The researchers compared the target and tracker signals to make the following measurements: (a) cross correlation, (b) gain ratio, (c) phase shift, and (d) average amplitude difference between the two signals (TTD). Results: Cross correlation measures between the target and tracker signals revealed improvements after 8 years of age until 15 to 20 years of age, when the correlation plateaus, followed by a decline in correlation at 40 to 45 years of age. These measures also showed a significant main effect of age group and target frequency. The trends and effects found in the cross correlation measures were found to be similar for the gain ratio and phase shift measures. Target frequency for lip movement was also found to be a significant main effect in the gain ratio measures. Phase shift measures also showed a significant main effect of target frequency for fundamental frequency of age group. The trends found in the cross correlation measures were also found in the TTD measures as amplitude variability was the lowest for adults between 17 and 45 years of age. Conclusion: Target-to-tracker signals improved with the young adult age group, but declined with the middle-aged group, indicating that aging affects articulatory visuomotor control. Relevance to the current work: This study provides support that aging impacts articulatory performance on visuomotor tracking tasks.

Becic, E., Dell, G. S., Bock, K., Garnsey, S. M., Kubose, T., \& Kramer, A. F. (2010). Driving impairs talking. Psychonomic Bulletin \& Review. 17(1), 15-21. doi: 10.3758/PBR.17.1.15

Objective: This study was designed to examine the effects of a simulated driving task on speech production, comprehension, and memory in older and younger adults. Method: One hundred ninety-two adults participated in this study. Half of these participants were considered as older adults $(M=70.7$ years $)$, and the other half were considered younger adults $(M=19.6$ years). 
Participants in this study were grouped into pairs and assigned roles. One adult was assigned the role of the driver, and the other adult was assigned the role of the passenger. Each participant completed questionnaires regarding their driving habits, and all older adults were highfunctioning and drove regularly. The participants were asked to perform three different block tasks: a speech-only task, a driving-only task, and a dual-task. The speech-only task required the driver to listen to a short narrative $(M=12.7$ seconds long) through headphones and to retell the story in their own words to the passenger within a 30-second time period. This task was done in the simulator with the car in park. The roles of storyteller and listener were then reversed. Between the two different speech blocks (speech-only and dual), each participant heard and retold four stories. The dual-task, however, only required dual activity from the driver. To assess memory, after the participants completed each block with the speech task, the driver and passenger were instructed to leave the simulator and to recall the stories told by their partner. The driving-only task required the drivers to navigate through an urban environment with busy intersections while also obeying the $30 \mathrm{mph}$ speed limit, remaining in the middle of their lane, stopping at stop signs, and crossing intersections safely. Results: Continuous-driving measures revealed that drivers had greater variability in velocity and staying in their lane in the drivingonly task compared to the dual-task. These results showed that participants slowed down and had less variability in velocity during the dual-task compared to the driving-only task, especially the older participants. Discrete driving measures revealed that the participants braked closer to the stop sign during the dual task compared to the driving-only task. These measures also revealed that the older adults braked farther away from stop signs, as well as required more time to cross intersections compared to the younger adult drivers. Speech-task performance was measured by accuracy of story retelling and memory performance, all of which were performed as a four-way mixed-mode ANOVA analysis. The results showed that the participants had more accurate story retelling during the speech-only task compared to the dual task, although the younger adults had more accurate story retell than the older participants. The accuracy of story retell in the drivers was less accurate than the passengers during the dual task, especially when the route demanded more from the participants' driving (e.g., through intersections). Measurements in the memory test performance revealed that the drivers had better recall of stories during the speech-only task than the dual task, whereas the passengers had good recall in both conditions. The results also showed that the driving demands of the route yielded no significant effects on recall. Older participants also showed poorer recall than the younger participants. Conclusion: As expected, driving can have a negative impact on speech production, comprehension, and memory as driving can take priority over the conversation. Story retell was negatively impacted by driving, especially during times of route difficulty. Relevance to the current work: This study provides evidence that speech is negatively affected during dual tasks such as driving. This study also analyzes how the cognitive work load in each of the tasks impacts the others' performance. 
Beede, K. E., Kass, S. J. (2006). Engrossed in conversation: The impact of cell phones on simulated driving performance. Accident Analysis \& Prevention. 38(2), 415-421. doi:10.1016/j.aap.2005.10.015

Objective: The purpose of this study was to examine how driving performance is affected when distracted by other cognitive-demanding tasks. With the rising popularity of cellular phones in the United States, drivers are more likely to talk on the phone while driving. Research has shown that the negative effects of talking on the phone while driving are a result of the two tasks competing for limited cognitive resources rather than the driver using one hand to hold the phone. Thus, this study was designed to see how the cognitive demands of talking on the phone while driving impacts driving performance. Method: Participants in this study included 36 student volunteers enrolled in psychology courses from the University of West Florida. These participants all had a valid driver's license, a median of six years of driving, and a median of 450 miles driven per month. Participants also were within the 20- to 53-year age range. Researchers used STISIM Drive software by Systems Technology Inc. to measure driving performance. Participants experienced the same driving scenarios and all wore headphones during the driving task. Two distraction conditions were incorporated in this study: a signal detection task and a telephone task. During the signal detection task, participants were asked to press the buttons on the side of the steering wheel to match the signal arrows appearing in the bottom right or left corner of the screen. These signals appeared randomly and the driving task continued regardless of the response. The telephone task involved participants conversing with pre-recorded questions and statements that were programmed to be played when the driver reached a certain location in the simulation. Participants performed this in two different talking conditions. Both conditions were similar as far as cognitive demand, and incorporated declarative questions and questions requiring mental imagery (e.g., "How do I get to the mall from your house?"). Both the telephone and signal detection tasks were involved to increase the cognitive demands of the study. Participants completed a questionnaire prior to the tasks to inform researchers of the participants' demographics, cellular phone usage, driving history, and driving behaviors. Four areas were examined to assess participants' driving performance: violations, driving maintenance, attention lapses, and reaction time. Prior to testing, researchers standardized the driving scenarios and had the participants complete a practice scenario that lasted 18 minutes. Results: The results from the questionnaire revealed driving behaviors such as accidents (67\%), traffic tickets $(M=1.7$ tickets), and cell phone usage ( $58 \%$ for dialing and $80 \%$ for conversing). The results from the signal detection task showed that the task had little impact on traffic violations, whether using a cell phone or not. The results from the telephone task revealed a negative impact on driving performance regarding traffic violations as participants committed more violations when conversing in a simulated cell phone conversation than in conditions without cell phone usage. The results of driving maintenance revealed an interaction effect between the telephone and signal detection task as participants' drove faster, stayed in their lane better, and changed lanes less frequently. The findings regarding attention lapses showed that both the telephone and signal detection tasks yielded an increased number of attention lapses committed while driving, with the telephone task showing a significant impact on attention. The telephone task also showed longer delays in reaction times while driving whereas the signal detection task did not have the same effects. Conclusion: The results of this study revealed that secondary tasks while driving can impact driving performance. Cell phone usage, in particular, was proven to show an increase in traffic violations and attention lapses committed. Thus, the 
sharing and competing demands of cognitive resources can interfere with each other when it comes to conversation and driving tasks. Relevance to the current work: This study provides evidence that driving performance is impacted when conversing on the telephone, but does not provide the speech aspects of it. It also gives implications of the limited cognitive resources during speech and motor tasks. This study also provides a framework of criteria that participants should meet in order to participate in the study.

Bergen, B., Medeiros-Ward, N., Wheeler, K., Drews, F. A., \& Strayer, D. L. (2013). The crosstalk hypothesis: Why language interferes with driving. Journal of Experimental Psychology: General, 142(1), 119-130. doi: 10.1037/a0028428

Objective: The purpose of this study was to determine the nature of interference when individuals perform two tasks at the same time. This study focuses on two theories: (1) that interference is domain-general, and (2) that the interference is domain-specific, which is also known as the crosstalk hypothesis. The domain-general interference theory suggests that the brain has limited attentional resources and that when two tasks are completed at the same time, more attentional resources are demanded and thus, performance in one or both tasks declines. The domain-specific interference theory suggests that the more similar the processing and performance of the two tasks (e.g., visual, motor, auditory, etc.), the more interference there will be which produces "crosstalk", resulting in impaired performance in one or both tasks. Method: The experiment had two versions: Participants in this study included 145 undergraduate students who participated in one of the two versions of the experiment. Fifty-two of these participants were excluded from the study due to low accuracy scores on the language task or motion sickness from the driving simulator. A total of 93 students participated in the study (50 in Version 1 and 43 in Version 2). Participants included 46 females and 47 males who met the following requirements: (a) ranged in age from 18 to $32(M=23.2)$, (b) had normal vision, (c) had no neurological disorders, (d) were native English speaker or spoke English fluently, and (e) had a valid driver's license. The driving simulator (PatrolSim) provided a 180-degree view of the driving environment, realistic scenes, and traffic conditions. Participants completed a 10minute training session prior to the experiment to help them adapt to the simulator. In the experiment, participants drove on a multilane freeway with a pace car that would brake at random intervals in the right lane, as well as other vehicles that were programmed to drive 5$10 \%$ faster than the pace car to mimic real-life traffic conditions. Driving performance was measured by following distance (average distance from the leading car) and braking reaction time. In both versions of the experiment, participants were presented with 32 prerecorded single sentences (half of the statements being true and the other half being false) in three out of the four language conditions used. Four different language conditions were used to observe the effects of language on driving: a motor condition with the sentences having motor content (e.g., "To close a jar, you turn the lid clockwise"), a visual condition with the sentences having visual content (e.g., "The letters on a stop sign are red"), an abstract condition with the sentences having vague visual or motor content (e.g., "The capital of Utah is Provo"), and a control condition in which participants heard and complied with two sentences, "Say the word false" and "Say the word true", each repeated 16 times. Version 1 of the experiment had participants respond to the sentences by stating whether they were true or false. Version 2 of the experiment had participants repeat the true statements and correct the false statements. Results: The results revealed no significant differences between the two versions on any of the measures. There was 
an increase in braking reaction time in the experimental language conditions compared to the control condition, although these differences were not significant. The results for following distance revealed language as a main effect as the differences were dependent on the language condition. The motor and visual conditions, for instance, had greater following distances than the other conditions. The only significant differences were between the visual condition and the abstract and control conditions. Other analyses revealed a difference between the motor and control conditions and the motor and abstract conditions, although it was not significant. The researchers also examined the variability of following distance and found significantly less variability in the abstract condition than in the visual or motor conditions. The results of the language task in Version 1 revealed a main effect of language condition on accuracy. The results of the individual language conditions showed a significant difference between the visual condition and the motor and abstract conditions; participants were most accurate in their responses in the visual condition compared to the other conditions. The accuracy measures in the experimental language conditions were similar to the measures in the control condition, indicating that driving performance was affected by the type of language, but that the language processing was not affected by the driving. The accuracy rate differences between the experimental conditions and the control condition showed that the visual and abstract sentences were the most affected sentence types, whereas the responses for the motor sentences were affected minimally by the dual-task condition. Conclusions: The results of braking reaction time in the three experimental language conditions provided support for the domain-general interference prediction as all three conditions interfered with vehicle control. The results of the following distance measures, however, provided support for the domain-specific interference prediction (or crosstalk hypothesis) as the visual condition results indicated a higher level of distraction than the other conditions due to the processing of the language content that was similar to the processes needed to drive a vehicle. The authors also found that the abstract language condition was the most unfavorable with regard to driving conditions as the driver is slower to respond to the driving environment. These results suggested that different types of language can have different effects on motor control and perceptual tasks. These findings also suggested that a code conflict can exist when an individual is using perceptual and motor types of language when concurrently engaged in perceptual or motor tasks. Relevance to the current work: This study focuses on the processing of different types of language and how these types can have different effects when driving, providing good insight into the types of language tasks that might be used in the current study.

Cao, S., Liu, Y. (2013). Concurrent processing of vehicle lane keeping and speech comprehension tasks. Accident Analysis \& Prevention. 46-54. doi:10.1016/j.aap.2013.04.038

Objective: The purpose of this study was to examine the effects of speech comprehension tasks on concurrent vehicle lane maintenance. The authors also investigated the mental workload in the dual-task interaction. Method: To improve experimental control from previous studies, the researchers in this study kept the driving speed constant and only examined one aspect of driving (i.e., lane keeping) that was designed to be difficult. The study also focused on speech comprehension performance. Participants in this study included 17 male and 7 female Mandarin Chinese speakers with an average age of 29.6 years. All participants had a valid driver's license, normal or corrected vision and audition, and at least $8,000 \mathrm{~km}$ of driving experience. The 
driving simulation was developed using Micro Saint Sharp and was used on a PC computer. Drivers were instructed to stay as close as possible to the center of their lane. The speech comprehension task was based on a previous study in which the participants listened to sentence pairs and determined whether or not the sentences had the same meaning by pressing buttons located on the steering wheel. Participants were instructed to give their answer as quickly as possible during the speech comprehension task. During the dual-task condition, drivers were instructed to give priority to the driving task and then complete the speech comprehension task. Participants were given a six-minute practice time in all the conditions prior to the experiment. They were also given feedback on their responses in the speech comprehension task during this practice session. The actual experiment lasted about 80 minutes and participants were given short breaks throughout the session. Results: The results revealed that vehicle speed had a significant effect on lane keeping performance when speeds were higher. The effects of the task condition yielded no significant effect on lane keeping performance. There was also no significant effect on steering wheel control in any of the task conditions, vehicle speeds, or interactions. The results of speech comprehension performance revealed no significant effect on reaction time in any of the task conditions, vehicle speeds, or interactions. Task condition had a significant effect on the rate of correct responses as the correct response rate was reduced in the dual-task condition compared to the single-task condition. Conclusion: Based on these results, the researchers concluded that secondary speech comprehension tasks may not immediately impact the primary task, but they do increase the mental workload, which causes a reduction in speech comprehension. The authors also concluded that dual-task conditions increase the potential risks of driving when performed for a longer period of time. Relevance to the current work: This study examined the mental workload required for simulated driving and speech comprehension in single-task conditions and dual-task conditions. This study also suggests that speech comprehension is reduced when other tasks are completed concurrently.

Drews, F. A., Pasupathi, M., \& Strayer, D. L. (2008). Passenger and cell phone conversations in simulated driving. Journal of Experimental Psychology: Applied, 14(4), 392-400. doi: $10.1037 / \mathrm{a} 0013119$

Objective: The purpose of this study was to examine and compare the mental demands of conversations with passengers in a vehicle and conversations on a cell phone while driving. To determine the amount of distraction in each task, the researchers studied three levels of driving performance: lane maintenance (i.e., staying in the center of the lane), traffic behaviors (i.e., speed and reaction times), and navigation. Method: Participants included 47 women and 49 men with normal vision, and a valid Utah driver's license; they were 18 to 49 years old $(M=20$ years old). The 96 participants were assigned into 48 pairs. The simulation included a 24-mile, two-lane freeway with irregular-flow driving conditions that would require the driver to pay more attention to the surrounding traffic. Participants were given a 15-minute adaptation sequence to help familiarize them with the simulation. Drivers were then randomly selected within a pair, with the other individual assigned as a passenger in the vehicle or as a cell phone user. The speakers were instructed to share a story that the driver had not heard before, while the drivers were instructed to follow the traffic rules, drive safely, and exit the freeway when arriving at a rest area. Drivers participated in a single-task condition in which the driver was instructed to just drive. In the dual-task condition, drivers were instructed to converse on a cell phone or talk to the passenger while driving. Results: The results of driving performance were 
analyzed with $t$ tests to examine the differences between the cell phone condition and the passenger condition. The results of lane maintenance revealed a significant difference as drivers drifted more often during cell phone conversations compared to conversations with a passenger. The results of traffic behaviors revealed no significant differences between the two conditions in driving speed, and a significant difference in keeping distance from the vehicle ahead as the drivers kept more distance between them and the next vehicle in the cell phone condition compared to the passenger condition. The navigation portion of the experiment was analyzed based on task completion. The results showed a difference between the two conditions as drivers were four times more likely to not complete the navigation task in the cell phone condition compared to drivers in the passenger condition. The researchers also compared four aspects of conversation between the two conditions: references to traffic, turn taking on the traffic topic, production rate, and complexity of speech. The results of the number of traffic references showed no differences by the driver, but a reliable difference by the non-driving participant as there were more references made in the passenger condition compared to the cell phone condition. The results of the turn taking analysis revealed that more than twice as many turns were made in the passenger condition compared to the cell phone condition when conversing about traffic. The results of production rate showed that drivers decreased their production rate in the passenger condition and increased their production rate in the cell phone condition when driving in moderately difficult driving conditions. The authors found no differences in production rate in the non-driving participant. The results of the driver and interlocutor's complexity of speech revealed a decrease in the number of syllables per word as the driving demands increased, but the differences did not reach significance. Conclusions: The authors concluded from the results that driving performance can differ based on contextual variables as some variables require more attention (e.g., passengers were able to support the driver by talking about the surrounding traffic whereas cell phone users were not able to provide that same support). The researchers also concluded that the differences between the two conditions could stem from differences in the structure of conversations when using a cell phone and when conversing with someone in person. Relevance to the current work: This study provides evidence of the allocation of attention in cognitively-demanding environments. This study also provides some preliminary evidence of how speech or language performance is affected in dualtask conditions, although most of the aims of the study were to analyze the effects on driving performance.

Dromey, C., \& Bates, E. (2005). Speech interactions with linguistic, cognitive, and visuomotor tasks. Journal of Speech, Language, and Hearing Research, 48(2), 295-305. doi:10.1044/1092-4388(2005/020)

Objective: The study was designed to examine the bidirectional interference between speech tasks and simultaneously performed linguistic, cognitive, and visuomotor tasks. Method: Ten males $(M=24.5$ years of age $)$ and ten females $(M=22.4$ years of age) sat in a sound booth with a head-mounted strain gauge system that measured the lip and jaw movements. A sound level meter was also used to measure vocal intensity. Participants performed seven tasks; four involved single-task conditions (speech-only, linguistic-only, cognitive-only, and visuomotoronly) to collect baseline performance; three involved completing the speech tasks while concurrently performing one of the other tasks. For the speech task, participants were instructed to repeat "Peter Piper would probably pick apples" 15 times. Measurements for this task 
included utterance duration, peak velocity for the second $/ \mathrm{p} /$ closure in the word Piper, correlation between upper lip and lower lip/jaw movements, spatiotemporal index (STI) for the lower lip/jaw movements, and SPL. For the linguistic task, participants were presented with eight or nine words on a computer screen and instructed to compose a grammatically correct sentence with the words. Measurements for this task were based on the number of grammatically correct sentences composed and latencies in the responses. For the cognitive task, participants completed math subtraction problems within 10-second intervals. Measurements for this task were based on the number of correct answers and latencies in responses. For the visuomotor task, participants were instructed to use the computer mouse to click on a moving object on the screen as often as possible. Measurements for this task were based on the number of accurate clicks in a given time. Results: The combined visuomotor task revealed a significant difference compared to the visuomotor-only condition as the combined condition resulted in a decrease in utterance duration. All the combined tasks resulted in a decrease in lower lip/jaw displacement compared to the single-task conditions, with the combined visuomotor task showing a significant decrease when compared to the speech-only condition. The results for STI revealed an increase in the combined conditions compared to the speech-only condition, with the combined linguistic task showing a significant increase in STI compared to the speech-only condition. The SPL results showed a significant increase in all the combined conditions compared to the speech-only condition. Latencies increased in the linguistic combined condition compared to the single-task condition, while performance in the other combined tasks remained unchanged compared to their single-task conditions. Conclusion: The combined linguistic task showed that the speakers had less consistent labial movements, spoke more loudly, and had lower linguistic scores compared to the linguistic-only condition. These changes in speech performance were found in the combined linguistic task, but not in the other combined tasks. These results support the functional distance hypothesis, although these results could be attributed to the task difficulty in the cognitive and visuomotor tasks. Based on these results, the researchers concluded that individuals' speaking performance changed when concurrently performing other activities. Relevance to the current work: This study provides quantitative data to show how speech is affected by and itself affects the performance of a concurrent task.

Dula, C. S., Martin, B. A., Fox, R. T., \& Leonard, R. L. (2011). Differing types of cellular phone conversations and dangerous driving. Accident Analysis \& Prevention, 43(1), 187-193. doi: 10.1016/j.aap2010.08.008

Objective: The purpose of this study was to determine the effects of driving performance from different types of conversations. Researchers, specifically, looked at dangerous driving behaviors during these conversations. These behaviors included speeding, traffic light violations, collisions, and crossing lanes. Method: Seventy-five undergraduate students in various psychology classes at a Southeastern university in the United States participated in this study. Ages ranged from 18 to $43(M=21.74)$ with 32 being female and 21 being male. Three conditions were used: a "no call" condition with 36 participants, a "mundane call" condition with 20 participants, and an "emotional call" condition with 15 participants. Participants were assigned to one of these groups based on the time slot they signed up for. A STISIM Drive computer simulated driving program was used in this study to assess different driving variables: number of speeding events, percent of time speeding during driving, number of collisions, number of center line crossings, and number of traffic light violations by running a red light. An 
emotional topic questionnaire was used to have participants write their feelings towards controversial topics such as gay marriage, abortion, and political party affiliation. Participants were given one practice to get used to the controls prior to the conversation. After 15 seconds of driving in the simulated task, a confederate from outside the room called the participant and engaged in a mundane conversation or an emotional conversation by arguing the opposite side of the participant's response. Participants in the "no call" condition did not receive any phone call and just drove through the course. Results: A Multivariate Analysis of Variance (MANOVA) was used to examine the effects on driving based on the call condition. Between-subject tests showed that the effect of the call condition was significant across all the dangerous driving behaviors besides the traffic light violations. The results for speeding occurrences revealed no significant difference between the "no call" and "mundane" conditions and a significant difference between the "mundane" and "emotional" conditions. The results for the percent of driving time spent speeding and the number of times the driver crossed the center line showed a significant difference between all the conditions as speeding time increased and more crossing violations occurred when the call intensity level increased. The results for the number of collisions showed significant differences between all call conditions except between the "no call" and "mundane" conditions. The results for traffic light violations yielded no significant differences which was concluded to be due to the limited number of traffic lights in the simulation. Conclusion: The hypothesis that dangerous driving behaviors are impacted by the type of conversation was supported in four out of the five variables as more dangerous driving behaviors were observed in the emotional conversation than the others. These results also show that mundane conversations impact some aspects of driving behavior, but that emotional conversations affects most to all driving behaviors. Therefore, more emotional conversations poses greater risks in driving than mundane conversations or no conversation at all as the cognitive load increases with more complex and emotional conversations. Relevance to the current work: This study not only supports the idea that driving performance is impacted by engaging in a conversation, but also that the type of conversation can impact driving behaviors. This study also shows that the cognitive load across the call conditions differs and that the increase of cognitive load results in more risky driving behaviors.

Kubose, T., Bock, K., Dell, G., Garnsey, S., Kramer, A., \& Mayhugh, J. (2006). The effects of speech production and speech comprehension on simulated driving performance. Applied Cognitive Psychology, 20, 43-64. doi:10.1002/acp.1164

Objective: This study was designed to assess what aspects of speech cause the most interference. This study specifically looked at speech production and speech comprehension and their effects on driving performance. Method: Researchers conducted a dual-task paradigm with the simulated driving as the primary task and the speech production or speech comprehension task as the secondary task. Two experiments were conducted with 48 participants in each experiment. Participants were native-English speakers from the University of Illinois. Experiment 1 had 27 male and 21 female participants with an average age of 21.73 years and an average driving experience of 5.13 years. Experiment 1 looked at continuous measures such as velocity and lane maintenance and how they are affected when simultaneously participating in speech production or speech comprehension tasks. Participants were asked to stay as close to 55 miles per hour as they could while driving. Experiment 2 had 20 male and 28 female participants with an average age of 20.2 years and an average driving experience of 3.96 years. Experiment 2 assessed the 
effects of speech production and speech comprehension on driving performance with regard to distance from the car ahead (headway) and lane maintenance. Participants were instructed to remain two seconds behind the vehicle in front of them. Both experiments were on a simulated two-lane highway with no turns or intersections and participants were instructed to try to remain in the center of their lane. Prior to the test, all participants completed a training session to make sure that they knew the buildings and locations on campus, and to also give them driving practice with the equipment. Three conditions were included in the study: drive-only, speech-only, and drive-speech which consisted of speech production and speech comprehension tasks. The speech production task required participants to name buildings on campus that were located in the specified direction in relation to the buildings presented. The speech comprehension task had participants verify whether the recorded statements from the speech production task were true or false by pushing buttons on the steering wheel. Results: The results of the speech tasks in both experiments showed no significant differences between the speech-only and drive-speech conditions as performance remained approximately $85 \%$ or more in all conditions. The results of driving performance in Experiment 1 showed an increase in the variability of velocity when under the drive-speech condition. The results from this experiment also showed that participants stayed in their lane and drove faster in the speech production task than the speech comprehension task. The results of driving performance in Experiment 2 showed that headway time in the drive-speech condition was greater and more variable than under single-task conditions. This experiment also showed that participants in the speech production task stayed in the center of their lane more often than in the drive-speech condition than the drive-only condition.

Conclusion: Due to the similar results between the two tasks, researchers concluded that both speech production and speech comprehension tasks play a role in interference on driving performance. With participants remaining in their lane more often in the dual-task condition rather than the single-task condition across both experiments, researchers also concluded that drivers can make adjustments and prioritize certain tasks to adapt to their driving condition. Relevance to current work: This study supports the findings in previous studies on driving performance and secondary speech tasks. This study also focuses on the cognitive work load in different speech tasks and how these tasks could limit the mental resources required for maintaining safe driving. A limitation in this study is that the speech tasks that participants perform are unnatural. This limitation justifies the current work as the speech tasks will be more naturalistic.

McDowd, J. M., \& Craik, F. I. (1988). Effects of aging and task difficulty on divided attention performance. Journal of Experimental Psychology: Human Perception and Performance, 14(2), 267-280.

Objective: The purpose of the study was to evaluate how task complexity interacts with age to impact performance under dual-task conditions. Method: Two experiments were conducted. The first experiment had 16 young adults $(M=19.4$ years of age $)$ and 16 older adults $(M=69.0$ years of age), who participated in two tasks (auditory and visual) at easy and hard levels. The participants performed each task separately first in single-task conditions, then combined in a dual-task condition. The easy auditory task had participants press a response key each time they heard a target word in a list of 12 words. The difficult auditory task had participants press a response key each time they heard a word that represented living things from 14 lists containing 12 words. The easy visual task required participants to press a key that corresponded with the 
position of an object that appeared on the monitor in front of them. The difficult visual task required participants to press a key that corresponded with the type of character (vowel, consonant, odd digit, and even digit) that appeared on the screen. Measures for all the tasks were based on response times. In experiment two, participants completed two visual tasks: a position task, and a faces task that was considered to be cognitively more difficult than the other task. Participants included a younger age group $(M=21.0$ years of age $)$ and an older age group $(M=$ 71.9 years of age for the position task, $M=67.3$ years of age for the faces task). Each task had three levels of difficulty. Each task was performed first in a single-task condition, then combined with an auditory task in a divided attention condition. Measures were based on response times as long as the divided attention task percentage remained above $75 \%$. Results: The results for experiment one's visual task revealed that more errors were made by the older adult participants under the dual-task condition compared to the younger adult participants, suggesting that age was a significant factor in dual-task performance. Task complexity also was a factor as reaction times increased for both age groups in both conditions. Task complexity also amplified decrements in dual-task performance for the older age group. Experiment two revealed mixed results. Age had a negative effect on dual-task performance as the difficulty of the faces task increased. The opposite results were found for the position task: as complexity increased, the effects of the divided attention condition decreased. Conclusion: Based on these results, the researchers concluded that older adults are negatively impacted in dual-task conditions more than younger adults, and that these effects are amplified when task complexity increases. The researchers also determined that divided attention conditions increase the complexity of the task. Relevance to the current work: This study provides support for the hypothesis that dual-task performance is better in younger individuals than in older individuals.

Medeiros-Ward, N., Cooper, J. M., \& Strayer, D. L. (2014). Hierarchical control and driving. Journal of Experimental Psychology: General, 143(3), 953-958. doi:10.1037/a0035097

Objective: The purpose of this study was to test the hierarchal control theory (HCT) and its role in cognitive performance when engaging in two tasks at once. HCT suggests outer and inner loops of control. The outer loop, for instance, selects the word to type while the inner loop executes the typing movements. The current research tested two aspects of this theory, the first being that performance that is dependent on the outer loop gets worse when less attention is given to the task, but gets better when more attention is on the task. The second aspect tested was that performance that is dependent on the inner loop does the opposite: when more attention is given to the task, the worse the performance is and the less attention given to the task, the better the performance is. Method: Participants in this study included 11 men and 16 women recruited from the University of Utah who all met the following requirements: (a) normal vision, (b) fluent in English, (c) between 19 and 43 years old $(M=25$ years old), (d) had a driver's license for 7 years, and (e) drove 10,000 miles on average per year. Participants performed tasks under three different levels of cognitive workload: single task, 0-back, and 2-back. In the single task condition, participants drove using the driving simulator which consisted of a straight threelane highway with the vehicle maintaining a $68 \mathrm{mph}$ speed the whole time. In the 0-back and 2back conditions, participants were presented with audible recordings of four randomized numbers from 0 to 9 while also driving the simulator. In the 0 -back condition, participants repeated out loud the last number they just heard. In the 2-back condition, participants repeated 
out loud the number that was presented two trials earlier. Prior to the experiment, participants completed a trial run of the simulation to help them adapt to it. Participants continued the warmup trial until they achieved at least $85 \%$ accuracy in all the conditions. Three different levels of wind entropy (low, medium, and high) were used as another distracting factor for participants to control lane maintenance. Results: The results showed an interaction between cognitive workload and wind condition with regard to lane position. As cognitive workload increased without wind, participants' lane position varied less, but when both cognitive workload and wind conditions increased, participants had more variability in their lane positioning. The results of lane positioning also revealed that there was no effect of cognitive workload and an effect of wind entropy. The researchers analyzed participants' performance on the three levels of cognitive workload and determined there was no effect of wind conditions and no interaction between the wind and cognitive workload. The results did reveal a main effect of cognitive workload as participants were more accurate in their responses on the 0-back task compared to the 2-back task. Conclusions: The study found that as cognitive workload increased in predictable driving conditions, lane maintenance increased as well. These findings were consistent with most other current research, but were inconsistent with the Adaptive Control of Thought-Rational model. These findings provided support for the HCT model. These findings also revealed the extent of the impact of cognitive workload on driving performance. Relevance to the current work: This study provided evidence of the impact of cognitive workload when performing two tasks at once.

Oomen, C. C., \& Postma, A. (2001). Effects of divided attention on the production of filled pauses and repetitions. Journal of Speech, Language, and Hearing Research, 44(5), 9971004. doi:10.1044/1092-4388(2001/078)

Objective: The focus of this study was to investigate how divided attention tasks impact disfluencies that are commonly produced in normal speech, specifically filled pauses and repetitions. Method: The participants in the study included 18 undergraduates ( 7 men and 11 women) from Utrecht University, all of whom were between 19 and 24 years old, were Dutch native speakers, had no experience in dual-task experiments, extemporaneous speaking, or piano playing, nor any history of speech, language, or hearing problems. The experiment included two tasks: a storytelling task and a blind tactile-form recognition task. The conditions in this study included two single-task conditions for each task, as well as a divided attention condition with both tasks being performed at once. The participants were given a practice trial before each condition. The storytelling task had participants look at five picture stories, with four of the stories consisting of six pictures and one consisting of eight pictures. In this task, participants were shown one picture at a time and were instructed to describe the picture accurately and to try to add up the pictures to make a story. The participants' responses were recorded and later transcribed and scored by two judges to ensure reliability. The scores were based on the type and number of filled pauses and repetitions produced during the task. In the blind tactile-form recognition task, the participants were given 30 seconds to explore sandpaper figures with their dominant hand. They were instructed to become familiar with the figure so that they would be able to recognize the figure when given four multiple-choice options. The participants explored a total of 11 figures and were given the multiple-choice options after exploring 2 figures. The researchers scored this task based on the percentage of correctly identified figures. Results: The participants produced more pauses and repetitions in the divided attention condition compared to 
the speech-only condition. The researchers analyzed the type of repetitions made in the divided attention condition and found that sound/part-word repetitions and word repetitions increased, but that phrase repetitions did not. The results of the tactile-form recognition task revealed a significant difference between the figures-only condition and divided attention condition as the average percentage of correctly identified figures was higher in the figures-only condition (77.44\%) compared to the divided attention condition $(60.33 \%)$. Conclusion: The divided attention condition resulted in more pauses and repetitions than in normal speech conditions. These results indicate that these disfluencies are not a result of limited attentional resources, but instead, are automatic reactions to speech planning difficulties, and that these speech planning difficulties tend to increase when individuals are performing a concurrent task. Relevance to the current work: This study illustrates how speech is affected under dual-task conditions, as well as how speech affects other tasks when performed simultaneously.

Pashler, H. \& Johnston, J. (1998). Attentional Limitations in Dual-Task Performance. In H. Pashler (Ed.), Attention (pp. 155-189). Hove, UK: Psychology Press.

Objective: The chapter provides an overview of different theories of dual-task interference including the bottleneck theory, the capacity sharing theory, and the crosstalk hypothesis. The chapter also provides evidence on dual-task performance limitations, particularly perceptual processing and central processing limitations. Content: The main theme the authors discuss in this chapter is that humans have limited cognitive resources and that when people are engaged in two tasks at the same time, performance suffers in one or both tasks due to these limited attentional resources. The authors provide explanations of these attentional limitations through the bottleneck theory, the capacity sharing theory, and the crosstalk hypothesis. The bottleneck theory proposes that certain cognitive processes are done in a sequence and must be carried out in this sequence, and thus, dual-task performance is limited because an individual cannot perform tasks at exactly the same time. The capacity sharing theory suggests that cognitive resources, though limited, are shared between the two tasks and that the rate or efficiency of the processing is dependent on the amount of resources available to the task at the time. The crosstalk hypothesis proposes that the more similar the processing needed to perform the two tasks are (e.g., visual, motor, auditory, etc.), the more interference there is, which results in a "crosstalk" effect with impaired performance in one or both tasks. Relevance to the current work: The theories on attentional limitations discussed in this chapter provide a framework for understanding potential sources of interference in dual-task performance.

Strayer, D. L., Cooper, J. M., Turrill, J., Coleman, J., Medeiros-Ward, N., \& Biondi F. (2013). Measuring cognitive distraction in the automobile. Retrieved from AAA Foundation for Traffic Safety: https://www.aaafoundation.org/sites/default/files/MeasuringCognitiveDistractions.pdf

Objective: This report presented three experiments that focused on constructing a framework to measure and understand cognitive distraction when driving a vehicle. Method: In each experiment, participants were instructed to perform under the following eight conditions: (1) a baseline single-task condition, (2) simultaneously listening to a radio, (3) simultaneously listening to a book tape, (4) simultaneously conversing with a passenger in the vehicle, (5) simultaneously conversing on a hand-held phone, (6) simultaneously conversing on a hands-free 
phone, (7) simultaneously using a text-to-speech email system, and (8) simultaneously performing the Operation Span (OSPAN) task using an auditory version. Participants in the first experiment performed these tasks without driving the simulator to act as a control group. Participants in the second experiment performed the tasks while also driving the simulator. Participants in the third experiment performed the tasks while also driving an instrumented vehicle in a residential area. The authors analyzed the following measures to assess mental workload: (a) reaction time, (b) response accuracy to a peripheral light detection task (DRT), (c) subjective workload measures from participants using the NASA Task Load Index, (d) Electroencephalographic (EEG) activity measures, (e) Event-Related Brain Potentials (ERPs) associated with the DRT task, and (f) primary-task measures obtained from the driving portions of the experiments. These measures were standardized to develop a 5-category rating system ( 0 being not distracting, and 5 being very distracting) which measured the amount of cognitive distraction in each task. Results: Based on the standardized scores, the researchers concluded that some activities are more cognitively distracting than others. The authors found the speechto-text email system to be the most distracting condition (Category 3) while listening to the radio or book tape were the least distracting (Category 1). Both conversation conditions (with a passenger and on a cell phone) were rated as Category 2 as far as cognitive distraction is concerned. From these conditions, the authors suggested that the two different types of conversations had minimal differences when the passenger could not help with the driving task. Conclusions: Based on these findings, the researchers established a correlation between impairments in driving performance and the cognitive workload required in some in-vehicle activities. This study also demonstrated that voice-based systems are more cognitively distracting than suggested. The experiments conducted also provided measures which allowed the researchers to establish a simple cognitive distraction scale, although further investigation is needed to include visual and manual sources of distraction in the analysis of these activities. Relevance to the current work: The report establishes different levels of cognitive distraction while driving a vehicle. The report also describes different conditions that could be viable distractors in the current study.

Strayer, D. L., \& Drews, F. A. (2004). Profiles in driver distraction: Effects of cell phone conversations on younger and older drivers. Human Factors, 46(4), 640-649.

Objective: The purpose of this study was to examine the hypothesis that driving performance in older adults declines faster than in younger drivers when participating in dual- tasks. Method: Participants in this study included 20 older adults ( $M=70$ years old) and 20 younger adults $(M=20$ years old), all of whom had normal vision, were in good health, and had a valid driver's license. The PatrolSim simulator was used in the study which included a multilane highway, programmed distractor vehicles that created a steady flow of traffic, a pace car in the right lane, and four different driving scenarios. The researchers measured driving performance by the average driving speed, following distance (average distance from the lead vehicle), brake onset time (the amount of time between when the pace car's brake lights turning on and when the participant brakes), and half-recovery time (the amount of time needed to recover $50 \%$ of the speed that was lost while braking). Prior to the experiment, participants completed questionnaires regarding their health status, psychometric information, and their input in interesting conversation topics. Participants also completed a 20-minute training session to help adapt them to the simulator. Two of the driving scenarios were used for the single-task condition 
while the other two scenarios were used for the dual-task condition. For the experiment, drivers were instructed to follow the pace car in the right lane. In the dual-task condition, drivers spoke with a research assistant using a hands-free cell phone about topics that were of interest to the driver. The phone call was made prior to the simulation so that no cell phone manipulation was needed. Results: Age was not a significant factor in driving performance in both the single- and dual-task conditions. Older drivers braked longer than the younger drivers, causing the older drivers to also have a longer recovery time after braking, although these results were not significant. The brake onset times for both the older and younger adults showed slower reaction times by $18 \%$ in the dual-task condition when compared to the single-task condition. The results of the following distance revealed that older drivers followed the leading car with greater distance than did the younger drivers. The results also showed that participants kept a greater following distance in the dual-task condition than in the single-task condition. The age and task condition interaction was found to not be significant as both age groups increased their following distance by $12 \%$ in the dual-task condition. The results of average driving speed revealed that younger adults drove faster than the older adults, although the age and task condition interaction was not significant. The main effect of single versus dual-task also yielded insignificant results. The results of the half-recovery time showed that older adults took longer to recover from their lost speed than the younger adults did. The researchers concluded that these results are due to the older adults having a longer braking time than the younger adults. Both age groups in the dual-task condition took $17 \%$ longer to recover their lost speed during braking. The authors also noted that six of the participants were in collisions while driving: two of the accidents occurring during the single-task condition ( 1 older adult and 1 younger adult) and four of the accidents occurring during the dual-task condition (1 older adult and 3 younger adults). Conclusions: Based on these findings, the authors concluded that talking on a hands-free cell phone influences driving performance equally for older and younger adult drivers. These results indicated that cell phone conversations distract drivers and that this interference is not only caused by the manipulation of a cell phone. Relevance to the current work: Age is a factor that is being considered in the current study. One limitation from this study was that the variety of topics used were not consistent and therefore, the amount of cognitive workload may have varied depending on the topic. This should be considered for the current study.

Strayer, D. L. \& Drews, F. A. (2007). Cell-phone-induced driver distraction. Current Directions in Psychological Science, 16(3), 128-131. doi:10.111/j.1467-8721.2007.00489.x

Objective: The purpose of the article was to examine if cell phone conversations impact driving performance due to inattention blindness, meaning that drivers fail to attend to the driving environment as some of their attention is on the cell phone conversation. The article provided four studies that were each conducted by the authors to test their hypothesis on inattention blindness. Method: Each of the four studies included a single-task condition, which involved driving without conversing on a cell phone, and a dual-task condition which involved driving and talking on a hands-free cell phone in a natural conversation. The driving simulator used in the studies provided realistic scenes and traffic conditions and also gave participants a full 180degree view of the road. The researchers also used a video-based eye-tracker device to monitor the participants' eye fixations. The first study compared the participants' performance between the two conditions by surprising the participants with a memory test at the end of the simulation. Participants were asked to identify whether or not the presented objects were in the simulation. 
The second study examined a reallocation policy: drivers can alter attention between the two tasks strategically by paying more attention to the cell phone conversation when less-relevant information was presented while also still processing highly-relevant information in the driving scene. The researchers placed 30 objects along the roadway and that varied with regard to safe driving (e.g., pedestrians, signs, cars, billboards, etc.). At the end of the simulation, participants completed a surprise memory test similar to the first study. This memory test, however, required participants to determine which objects were in the simulation among other objects that were not presented in the simulation. Participants also rated the objects as far as their relevance to safe driving. The third study measured and analyzed the drivers' brain activity elicited by events that happened during the simulation. The authors used the P300 component's amplitude recordings from the brain activity waves to determine how much attention was allocated to the event (e.g., a larger amplitude indicates superior memory performance) as well as the level of difficulty of the task (e.g., a smaller amplitude indicates increased task demands). Participants drove on a simulated multilane freeway while following a car that would brake at random times. The researchers then compared the amplitude of the P300 component to determine whether the driving impairments were caused by impaired retrieval of information at the time of the memory test or the initial encoding of objects while driving. The fourth study compared the demands of two different types of conversations commonly used in the vehicle: conversation on a cell phone and a conversation with someone in the passenger seat. Participants drove on a multilane freeway and were instructed to follow the given directions to reach a location while also conversing on the phone or with the passenger. Results: The results of the memory tests in the first study revealed that participants recognized objects more than twice as much in the singletask condition compared to the dual-task condition, even when participants' eyes were fixated on the objects for the same duration. The findings were consistent in the second study as the researchers found significant differences in the memory test between the two conditions. The ratings revealed no association between memory and traffic relevance in both conditions, indicating that drivers do not reallocate attention while driving and talking on a cell phone. The results of the third study revealed that the amplitude of the P300 component decreased by $50 \%$ in the dual-task condition compared to the single-task condition, suggesting that the drivers using the cell phone did not see the objects in the simulation since they did not encode the information as well when distracted by a cell phone conversation. The results of the fourth study revealed that $88 \%$ of the drivers conversing with the passenger navigated to the location successfully, while only $50 \%$ of the drivers conversing on the cell phone made it to the correct location successfully. Conclusions: The results of the first and second studies were consistent with the inattention blindness hypothesis as participants remembered objects in the driving environment less often when conversing on a cell phone compared to driving in isolation, even when the drivers' eyes were fixated on the objects for the same duration between the two conditions. The authors concluded that the cell phone conversation distracted the drivers from the external environment, which resulted in less attention on their driving performance and their driving environment. The results of the third study indicated that drivers do not react as well to the driving environment when conversing on a cell phone, because the demands of the cell phone conversation required some attention to divert from the driving situation to the cell phone conversation. Based on the findings in the fourth study, the researchers concluded that the demands in conversations with a passenger were less than the demands in conversations on a cell phone because passengers responded, paused, and helped the driver based on the traffic conditions. Based on all the studies, the researchers concluded that cell phone conversations 
demand enough attention from drivers to create an inattention blindness to some objects in the driving environment, creating risky driving situations. Relevance to the current work: This study provides evidence that talking on a cell phone while driving demands some attention to divert from the driving task to the talking task. The study, however, only studies the impact on driving performance and not the impact on the conversation.

Strayer, D. L. \& Johnston, W. A. (2001). Driven to distraction: Dual-task studies of simulated driving and conversing on a cellular telephone. Psychological Science, 12(6), 462-266. doi:10.1111/1467-9280.00386

Objective: The purpose of this study was to determine how conversations on cellphones interfere with driving performance. Researchers also wanted to determine the nature of the interference, whether it be due to peripheral-interference (i.e. holding the phone) or attentional interference. Method: Researchers conducted two experiments to determine the nature of the interference. The focus of the first experiment was to contrast the effects between handheld and free-hand cellphone conversations during a simulated driving task by having participants perform a tracking task. A control group was included in the study and had the participants listen to the radio while performing the tracking task. Participants in this study included 48 undergraduate students (half male, half female) from the University of Utah. These students had normal vision, ranged in age from 18 to $30(M=21.3)$, and were randomly assigned to the three groups (radio control, handheld phone, and hands-free phone). They were instructed to keep a cursor on the moving target and to also press a "brake button" when the light changed from green to red. Three phases were implemented in this experiment: a seven-minute warm-up phase, a single-task phase with just the tracking task, and a dual-task phase with both the tracking task and conversation. The radio control group was instructed to choose a radio broadcast to listen to during the dual-task experiment portion. An additional control condition was included with 20 undergraduate students from the University of Utah with the same credentials as in the original experiment. The task for this group was similar to the radio control group, but instead had the participants listen to an audio-book tape. The participants then answered 10 multiple-choice questions at the end of the study to determine how well the participants attended to the book. The second experiment focused on determining cell-phone interference on driving. Participants included 24 undergraduate (12 female, 12 male) students from the University of Utah within the age range from 18 to $26(M=20.5)$ and with normal vision. These students participated in a simulated driving task on an easy and difficult course. Participants were given a warm-up trial to adapt to the simulator and then drove both courses without using a cellphone. Participants then performed two different dual-tasks in both courses. One dual-task had the participants repeat words that the experimenter presented to them into a hand-held phone while driving. The second dual-task had the participants generate a different word that began with the last letter of the word presented by the experimenter. Results: The results from the first experiment with the tracking task revealed that participants missed the simulated traffic signals twice as much when talking on a cellphone than in the single-task. The results also showed that the reaction times for the dual-task condition was slower than the singletask condition. The results from the radio control group and the additional control group yielded unreliable data between the single- and dual-task conditions, but showed an increase of missed traffic signals in the cell-phone group than in the control groups. Researchers also found no significant differences in reaction times between the hands-free device and the hand-held phone. 
The results from the second experiment showed that the word-generation dual-task condition caused an increase in tracking errors than the repetition dual-task condition. The repetition dualtask condition did not yield significant differences when compared to the single-task condition. Conclusion: Based on the results from the first experiment, researchers concluded that both hands-free and hand-held phones impact driving performance and therefore, peripheral factors such as holding the phone does not contribute to interference while driving. Instead, the actual conversation caused an increase in missed traffic signals and delayed reactions to traffic signals. Based on the results from the second experiment, researchers concluded that more attention was required for generating new words than in repeating the same words as tracking performance was impacted more when participants were generating new words. These results verify previous literature with regard to the cellphone conversation requires more attentional demands than holding the phone and that these demands require the attention to divert from driving performance. Relevance to the current work: This study looked at the cognitive demands between a motor task and speech tasks and how the tasks interfered with one another. The limitations of this study is that the speech tasks were not realistic which justifies the current work.

Strayer, D. L., Watson, J. M., \& Drews, F. A. (2010). Cognitive distraction while multitasking in the automobile. In B. Ross (Ed.), The Psychology of Learning and Motivation (Vol. 54, pp. 29-58). Burlington: Elsevier Inc.

Objective: This chapter serves as a foundation to understanding the sources and risks of driver distraction, particularly, how cell phone conversations impair driving performance. Content: The authors discuss how cell phone conversations rely on similar cognitive sources that are also used when driving and that because of this, drivers can have inattention blindness when talking on a phone. The authors demonstrate through a number of studies how cell phone conversations differ from conversations in the vehicle with a passenger. They also discuss how a small portion of people (supertaskers) can perform under dual-task conditions without any impairment. Relevance to the current work: The sources and reasons of driver distraction when using a cell phone discussed in this chapter provides a framework to understand the division of attention while performing two tasks at the same time. This chapter also provides evidence that driving performance is impacted when talking on a cell phone, but lacks evidence in how driving impacts speech performance.

\section{Wickens, C. D. (1981). Processing resources in attention, dual task performance, and workload assessment (No. EPL-81-3/0NR-81-3). Champaign, IL: University of Illinois at Urbana- Champaign.}

Objective: The report outlines different theories of divided attention including structural and capacity theories, and how these theories differ. Content: The author provides an overview of structural theories and explains how differences in task structure or processing stages can affect dual-task performance. The author specifically raises questions about these structural theories, such as the bottleneck theory, but acknowledges that the theory attributes longer processing time to task difficulty, an idea commonly found in capacity theories. The author also overviewed capacity theories, explaining that when concurrently performing two tasks at once, attentional resources are limited and as a result, performance suffers in one of the tasks when the other task 
demands more attentional resources. The author then explains the time-sharing model: that attention is forced to rapidly alternate between two tasks when participating in divided attention tasks, causing both tasks to have similar processes and end results.

Relevance to the current work: The report discusses theories of divided attention, providing indepth information on the processes of attention under dual-task conditions. 


\section{APPENDIX B: Informed Consent}

Consent to be a Research Subject

\section{Introduction}

This research study is being conducted by Dr. Christopher Dromey of the Department of Communication Disorders at Brigham Young University to determine how driving and talking simultaneously impact each other, as well as how age and gender are factors in performing multiple tasks at once. Katy Glenn and Kelsey Simmons, who are both graduate students studying speech-language pathology, will assist with this research. You were invited to participate because you are a native speaker of English, with no history of speech, language, or hearing disorders.

\section{Procedures}

If you agree to participate in this research study, the following will occur:

- the study will take place at the BYU John Taylor Building in Room 110 at a time convenient for you

- you will be given 15 minutes to practice and become familiar with a computer-based driving simulator

- you will be given a list of topics and asked to select 10 topics that are most interesting to you; during the experiment you will be asked to speak for approximately 60 seconds about one or more of those topics

- you will be asked to drive the simulator for 15 minutes

- separate from the driving task, you will be asked to answer 3 questions presented by the researcher

- you will then be invited to answer questions or participate in a conversation using a cell phone or with a passenger, all while driving at the same time

- you will be compensated with $\$ 10.00$ in cash for your time at the end of the study

- total time commitment will be 1 hour

\section{Risks/Discomforts}

There no known risks of participation in this study; however, it is possible that you may experience mild fatigue by the end of the experiment. The researchers will provide you with a break whenever you need one during the study.

\section{Benefits}

There will be no direct benefits to you. It is hoped, however, that through your participation researchers may learn about attentional processes and may be able to eventually assist speechlanguage pathologists in improving their therapy techniques.

\section{Confidentiality}

The research data will be kept with subject codes instead of names on a password protected computer and only the researcher will have access to the data. At the conclusion of the study, the non-identifiable data will be kept in the researcher's locked lab. 


\section{Compensation}

As a token of our appreciation, you will receive $\$ 10.00$ for your participation; compensation will not be prorated.

\section{Participation}

Participation in this research study is voluntary. You have the right to withdraw at any time or refuse to participate entirely.

\section{Questions about the Research}

If you have questions regarding this study, you may contact Dr. Christopher Dromey at dromey@byu.edu or at 801-422-6461 for further information.

\section{Questions about Your Rights as Research Participants}

If you have questions regarding your rights as a research participant contact IRB Administrator at (801) 422-1461; A-285 ASB, Brigham Young University, Provo, UT 84602; irb@byu.edu.

\section{Statement of Consent}

I have read, understood, and received a copy of the above consent and desire of my own free will to participate in this study.

Name (Printed): Signature: Date: 


\section{APPENDIX C: Monologue Topics}

\section{Personal}

- Would you quit if your values did not match your employer?

- If you could be rich, famous, or influential, which would you choose and why?

- How would you define faith?

- How do you define wealth?

- Do you believe people make happiness or stumble across it?

- Which is more important, talent or hard work?

- Are you an introvert or an extrovert? What are the pros and cons of each?

Media

- Are antidrug and antismoking ads effective?

- What video game would you like to redesign?

- Do social media campaigns stimulate real change?

- Should people be allowed to obscure their identities online?

- Is TV stronger than ever, or becoming obsolete?

- What ideas do you have for a reality show?

- What is your opinion about violence on television and in video games?

- What artists of today are destined for the Rock and Roll Hall of Fame?

Generations

- What is the difference between your generation and my generation and why?

- Is your generation more self-centered than earlier generations?

- Are young people generally more selfish than their parents and grandparents?

- How will our current culture be remembered in history books?

- Do children today have good manners?

- Does age make you more aware of and caring for others?

- Should adults try to teach young people lessons or should they leave them alone to find out about things themselves?

- Should parents continue to financially support their children after the children are 18 ?

- Is modern culture ruining childhood?

\section{Local Issues}

- If you could expand the trax system, what changes would you make?

- What do you see as the pros and cons of the proposed rebuilding of the Salt Lake Airport?

- Is it important to shop at locally owned businesses?

- What could be done about Salt Lake's homeless population?

- What are the pros and cons of the Sugarhouse trolley? 


\section{Social}

- What has caused the obesity epidemic in America?

- Should people get plastic surgery?

- Should rich people have to pay more taxes?

- What is your opinion about cloning?

- What are the ethical implications of eating meat?

- Are children of illegal immigrants entitled to a public education?

- Should welfare recipients be required to take drug tests?

- If you were a philanthropist, what groups would you finance and why?

- When should juvenile offenders receive life sentences?

- Should women soldiers be in combat?

- What is your opinion about legalizing marijuana?

- Are we losing the art of listening?

- Do attractive people have advantages others don't?

- What are the most important changes in the world since the year 2000 ?

Education and Related

- Is online learning as good as face-to-face learning?

- How necessary is a college education?

- Should cash-strapped schools cut arts education?

- Should guns be permitted on college campuses?

- What do you think about home-schooling vs. public vs. private school?

- How would you make over the university system?

- Whose fault is it if a child is failing in school?

- Should parents/grandparents give cash rewards to kids for good test scores?

- Should university students be required to take drug tests?

- Should junk foods and soda-pop be sold in elementary school or high school vending machines?

- How well do you think standardized tests measure people's abilities? 